\title{
A CALCULUS FOR PLUMBING APPLIED TO THE TOPOLOGY OF COMPLEX SURFACE SINGULARITIES AND DEGENERATING COMPLEX CURVES \\ BY
}

\author{
WALTER D. NEUMANN ${ }^{1}$
}

\begin{abstract}
Any graph-manifold can be obtained by plumbing according to some plumbing graph $\Gamma$. A calculus for plumbing which includes normal forms for such graphs is developed. This is applied to answer several questions about the topology of normal complex surface singularities and analytic families of complex curves. For instance it is shown that the topology of the minimal resolution of a normal complex surface singularity is determined by the link of the singularity and even by its fundamental group if the singularity is not a cyclic quotient singularity or a cusp singularity.
\end{abstract}

In this paper we describe a calculus for plumbed manifolds which lets one algorithmically determine when the oriented 3-manifolds $M\left(\Gamma_{1}\right)$ and $M\left(\Gamma_{2}\right)$ obtained by plumbing according to two graphs $\Gamma_{1}$ and $\Gamma_{2}$ are homeomorphic (3-manifolds are oriented 3-manifolds throughout this paper, and homeomorphisms of 3-manifolds are orientation preserving). We then apply the calculus to answer several questions about the topology of isolated singularities of complex surfaces and one-parameter families of complex curves. These results are described below.

Since the class of 3-manifolds obtainable by plumbing is precisely the class of graph-manifolds, which were classified, with minor exceptions, by Waldhausen [24], a calculus for plumbing is in some sense implicit in Waldhausen's work. Moreover, it has been known for some time that the calculus can be put in a form like the one given here, but the details have never appeared in the literature. A related calculus for plumbing trees has been worked by Bonahon and Siebenmann [1] in order to classify their "algebraic knots".

We describe the calculus in greater generality than is needed for the present applications, since this involves minimal extra work, and the calculus is needed elsewhere ([4], [14], and [15]). In particular, in an appendix we describe two generalizations of it.

The calculus consists of a collection of moves one can do to a plumbing graph $\Gamma$ without altering the plumbed manifold $M(\Gamma)$. To see these moves are sufficient, we describe how they can be used to reduce any graph to a normal form which is

Received by the editors April 22, 1980.

1980 Mathematics Subject Classification. Primary 32C45, 57N10.

Key words and phrases. Complex surface singularity, resolution, degenerating family of curves, graph manifold, plumbing.

${ }^{1}$ Research partially supported by the NSF. The hospitality of the SFB 40 in Bonn during the preparation of the paper is gratefully acknowledged. 
uniquely determined by the plumbed manifold. Our normal form is not the most elegant one possible, it is chosen the way it is (based first on predominantly negative euler number weights and secondly on as small graphs as possible, except that Seifert manifolds are given their natural "star-shaped" plumbing graphs) in order to expedite the proofs. The calculus is described in $\$ \S 1-7$.

The applications to complex surfaces and curves are proved in $\$ \S 8-10$. We first describe our results for singularities. Let $p$ be an isolated normal singularity of a complex surface $V$. The boundary $M^{3}=M^{3}(V, p)$ of a closed regular neighborhood of $p$ in $V$ is a closed orientable 3-manifold called the link of the singularity $(V, p)$. We say briefly: $M^{3}$ is a singularity link.

THEOREM 1. Singularity links are irreducible 3-manifolds.

This answers affirmatively Problem 3.28 of [10]. Our results also imply that the only non-sufficiently-large singularity links are the obvious ones: Seifert fibrations over $S^{2}$ with at most three exceptional fibers.

The following theorem answers affirmatively a question which I heard from A. Durfee, though it has apparently been asked by others too. It supports the conjecture that any deformation of a hypersurface singularity $(V, p)$ which preserves its Milnor number arises from a deformation of the resolution.

THEOREM 2. The topology of the minimal good resolution of the singularity $(V, p)$ is determined by the (oriented homeomorphism type of the) link $M^{3}$.

Using the next theorem, we get a much stronger result, as described in the subsequent corollary.

THEOREM 3. If $M^{3}$ is a singularity link then $-M^{3}$ ( $M^{3}$ with reversed orientation) is also a singularity link if and only if $M^{3}$ is either a lens space $L(p, q)$ with $p>1$ or a torus bundle over the circle whose monodromy has trace $\geqslant 3$. This holds if and only if the resolution graph is respectively a straight line graph or a cyclic graph.

COROLlary 4. If $M^{3}$ is a singularity link of the singularity $(V, p)$ and $M^{3}$ is not as described in Theorem 3, then $M^{3}$, and hence also the topology of the minimal good resolution of $(V, p)$, is determined by $\pi_{1}\left(M^{3}\right)$.

Note that Theorem 3 also implies that a singularity link $M^{3}$ has an orientation reversing homeomorphism, that is $M^{3} \cong-M^{3}$, only if $M^{3}$ is as in Theorem 3 . The singularity links of Theorem 3 do not all satisfy $M^{3} \cong-M^{3}$; those that do are easily determined using $\$ 7$ of this paper.

The next theorem answers a question of Wagreich [23]. It is practically, but not quite, a corollary of Theorem 3 .

THEOREM 5. If the Seifert manifold $M$ is a singularity link then its resolution graph is star-shaped.

The following corollary follows from [16, Theorem 5.2].

COROLlary 6. The Seifert manifold $M$ is a singularity link if and only if it has a Seifert fibration with orientable base such that the euler number of this fibration in the sense of [16] is negative. 
A consequence of Theorem 5 is that, if the singularity link $M$ of $(V, p)$ is a Seifert manifold, then the analytic structure of $(V, p)$ can be altered until $(V, p)$ admits a $\mathbf{C}^{*}$-action. $(V, p)$ itself need not admit such an action, in fact examples are known which show that this alteration of analytic structure cannot always be done by an analytic deformation.

Using different methods we show that very few singularity links can be fibered over the circle:

THEOREM 7. The singularity link $M^{3}$ fibers over $S^{1}$ if and only if $M^{3}$ is a torus bundle whose monodromy $A \in \operatorname{SL}(2, Z)$ satisfies either $\operatorname{trace}(A) \geqslant 3$ or $A$ is conjugate to $\left(\begin{array}{ll}1 & 0 \\ b & 1\end{array}\right)$ with $b>0$.

We obtain similar results for 1-parameter degenerating families of closed complex curves. We can write such a family as $\pi: W \rightarrow D$, where $W$ is a nonsingular complex surface, $D$ is an open disc about the origin in $\mathbf{C}, \boldsymbol{\pi}$ is a proper holomorphic map whose only singular value is $0 \in D$. Thus away from $\pi^{-1}(0), \pi$ is topologically a fibration with a fiber a closed orientable real surface $F$. We say the family is good if the only singularities of the singular fiber $\pi^{-1}(0)$ are normal crossings. This can always be achieved by blowing up in $W$. A minimal good family is a good family which does not result from blowing up a simpler good family. If $S^{1} \subset D$ is a small circle about the origin, then $M=\pi^{-1}\left(S^{1}\right)$ is the boundary of a regular neighborhood of $\pi^{-1}(0)$, and is called the link of the family.

The collection of data consisting of the genera of the irreducible components of $\pi^{-1}(0)$, their intersection behavior and their multiplicities, is called the numerical type of the family. It is well known that the numerical type determines both the topology of the pair $\left(W, \pi^{-1}(0)\right)$ and the euler number of the nonsingular fiber $F$. In particular it determines the link $M$, though it does not necessarily determine the topology of the fibration $\pi \mid M: M \rightarrow S^{1}$.

THEOREM 8. If $M$ is the link of a minimal good family as above, then $M$ determines the topology of the pair $\left(W, \pi^{-1}(0)\right)$. Moreover $M$ together with the euler number of a nonsingular fiber $F$ determines the numerical type of the family.

THEOREM 9. Both $M$ and $-M$ are links of families of curves if and only if $M$ is $a$ Seifert manifold with orientable base and the euler number of the Seifert fibration in the sense of [16] is zero.

COROLlaRY 10. If $M$ is a link of a family of curves which is not as in Theorem 9, then $\pi_{1}(M)$ determines (the oriented homeomorphism type of) $M$, and hence the topology of $\left(W, \pi^{-1}(0)\right)$.

It is very plausible that the link $M$ of $\pi: W \rightarrow D$ together with the fibration $\pi \mid M$ : $M \rightarrow S^{1}$ determines $\pi: W \rightarrow D$ up to homeomorphism (that is, the monodromy of $\pi$ determines the topological type of $\pi$ ), but I do not have a complete proof of this.

Theorem 9 implies that a link $M$ of a family which satisfies $M \cong-M$ must be Seifert fibered with euler number zero. The Seifert fibrations with $M \cong-M$ are determined in [16]; see also $\$ 7$ below. 
Two simple but amusing corollaries of Theorems 3 and 9 above are the following.

COROLlary 11. A complex surface $V$ is topologically the suspension of a closed 3-manifold, if and only if it is homeomorphic to an Inoue surface [8].

ADDED IN PROOF. "Homeomorphic" can be replaced here by "analytically isomorphic", by recent work of I. Nakamura.

COROLlARY 12. If a complex surface fibers over $\mathbf{C} P^{1}$ with exactly two singular fibers then the geometric monodromy has finite order.

Indeed, in Corollary 11 the two singularities of $V$ must have links of torus bundle $M^{3}$ and $-M^{3}$ respectively, with $M^{3}$ as in Theorem 3 , since the lens space case would lead, by a branched covering, to a complex structure on $S^{4}$, which is impossible. For any torus bundle $M^{3}$ as in Theorem 3, Inoue gave a complex structure on the suspension of $M^{3}$. Similarly, Corollary 12 follows from Theorem 9 and the fact that any fibration of a Seifert manifold as in Theorem 9 over $S^{1}$ has finite monodromy [2]. A set of examples for Corollary 12, which gives all homeomorphsm types of such examples with minimal good singular fibers, is given by taking fiber products $F \times_{\mathbf{Z} / n} \mathbf{C} P^{1}$, where $(F, \mathbf{Z} / n)$ is a $\mathbf{Z} / n$-action on a complex curve, and resolving the singularities, which are cyclic quotient singularities.

1. Plumbing 3-manifolds. In order to describe the calculus expediently, we will need a somewhat more general concept of plumbing than is usually considered in the literature. First some terminology.

If $F$ is a compact surface $g(F)$ will be its genus, with the convention that $g(F)<0$ if $F$ is nonorientable. Thus $g\left(\mathbf{R} P^{2}\right)=g(M b)=-1, g(k l)=-2$, and so on, where $M b$ is the Möbius band and $k l$ is the klein bottle.

If $A=\left(\begin{array}{ll}a & b \\ c & d\end{array}\right) \in \operatorname{SL}(2, \mathbf{Z})$, we denote also by $A$ the diffeomorphism of the torus $T^{2}=S^{1} \times S^{1}$ given by $A: S^{1} \times S^{1} \rightarrow S^{1} \times S^{1}, A\left(t_{1}, t_{2}\right)=\left(t_{1}^{a} t_{2}^{b}, t_{1}^{c} t_{2}^{d}\right)$.

A connected closed plumbing graph is a finite connected graph $\Gamma$ whose vertices and edges are assigned weights as follows:

(i) each vertex $i$ carries two integer weights $e_{i}$ and $g_{i}$;

(ii) each edge of $\Gamma$ is assigned a sign + or - .

We allow $\Gamma$ to have multiple edges and loops-that is several edges from one vertex to another or edges connecting a vertex to itself.

Given such a plumbing graph $\Gamma$, the plumbed 3-manifold $M(\Gamma)$ is constructed as follows. For any vertex $i$ of $\Gamma$ with weights $g_{i}$ and $e_{i}$, let $d_{i}$ be the degree of the vertex, that is the number of incident edges at this vertex (with loops at the vertex counted twice). Let $F_{i}$ be a compact surface of genus $g_{i}$ with $d_{i}$ boundary components. Let $E_{i} \rightarrow F_{i}$ be a circle bundle with oriented total space $E_{i}$, with a chosen trivialization of $E_{i} \mid \partial F_{i}$, and with euler number $e_{i}$ (this is the cross-section obstruction, and is well defined once the trivialization $E_{i} \mid \partial F_{i}$ is chosen). If $g_{i}>0$ we choose an orientation on $F_{i}$ and the trivialization of $E_{i} \mid \partial F_{i}$ is assumed compatible with this orientation. $M(\Gamma)$ is pasted together from the $E_{i}$ as follows: whenever vertices $i$ and $j$ are connected by a $(+)$-edge in $\Gamma$, we paste a boundary component 
$S^{1} \times S^{1}$ of $E_{i}$ to a boundary component $S^{1} \times S^{1}$ of $E_{j}$ by the map $J=\left(\begin{array}{l}0 \\ 1\end{array}\right)$ : $S^{1} \times S^{1} \rightarrow S^{1} \times S^{1}$; for a (-)-edge we do similarly using $-J=\left(\begin{array}{cc}0-1 \\ -1\end{array}\right): S^{1} \times$ $S^{1} \rightarrow S^{1} \times S^{1}$. Thus base and fiber coordinates are exchanged in the pasting and for a (-)-edge the orientations of both are also reversed. The pasting maps reverse orientation, so $M(\Gamma)$ inherits compatible orientations from all its pieces $E_{i}$.

We shall also allow disconnected plumbing graphs, with the following convention: if $\Gamma=\Gamma_{1}+\Gamma_{2}$ is the disjoint union of plumbing graphs $\Gamma_{1}$ and $\Gamma_{2}$, then $M(\Gamma)$ is the connected sum $M(\Gamma)=M\left(\Gamma_{1}\right) \# M\left(\Gamma_{2}\right)$. Finally, to avoid trivial special cases, it is convenient to allow the empty graph, with the convention that $M(\varnothing)=S^{3}$.

We also wish to describe manifolds with boundary by plumbing. This is done by assigning to each vertex $i$ an additional integral weight $r_{i} \geqslant 0$, and when plumbing, we remove $r_{i}$ open discs from the base space $F_{i}$ of the bundle $E_{i}$ being plumbed, creating $r_{i}$ additional torus boundary components on $E_{i}$, to which we paste nothing during plumbing, so they remain as boundary components of the plumbed manifold $M(\Gamma)$.

Notation. We write the weights as a vertex $i$ as

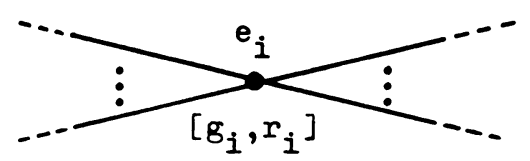

enclosing in square brackets the weights which refer to the topology of the base $F_{i}$ of the bundle being plumbed. If $r_{i}$ is zero we may omit it: if both $g_{i}$ and $r_{i}$ are zero we may omit both. If $r_{i} \neq 0$ then the cross-section obstruction $e_{i}$ vanishes and should be omitted, but for convenience we notice the convention: if an $e_{i}$-weight is written at a vertex with $r_{i} \neq 0$ it should be disregarded.

Thus a typical plumbing graph might look like the following

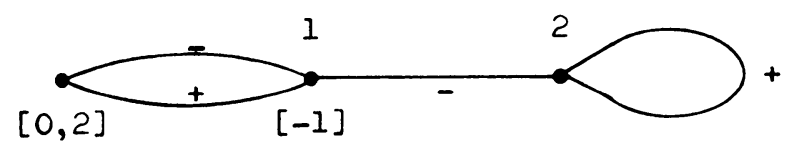

which is "shorthand" for

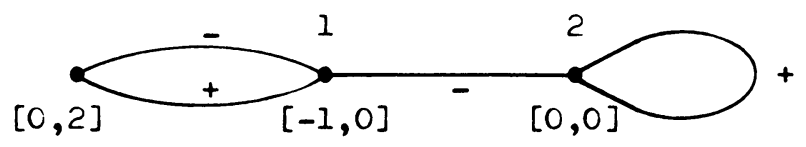

When drawing subgraphs of $\Gamma$, we always show all edges at the vertices drawn. The notation

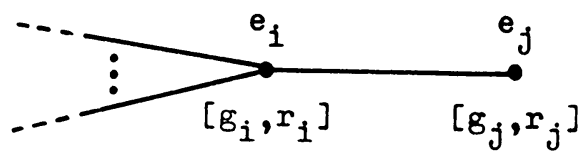

is meant to suggest that, in addition to the edge from $i$ to $j$, vertex $i$ may have any number (possibly zero) of additional incident edges. 
Terminology. A closed plumbing graph $\Gamma$ is one with all $r_{i}$ equal to zero, so $M(\Gamma)$ is a closed manifold. An orientable plumbing graph is one with all $g_{i} \geqslant 0$.

$M_{1} \cong M_{2}$ will always mean $M_{1}$ is diffeomorphic to $M_{2}$ preserving orientation. $M$ with reversed orientation is denoted $-M$ and $M_{1}+M_{2}$ means disjoint union.

2. The calculus. We shall need the following semigroup structure on $\mathbf{Z}$. If $F_{i}$ is a surface of genus $g_{i}$ for $i=1,2$, then we denote $g_{1} \# g_{2}=g\left(F_{1} \# F_{2}\right)$. Thus

$$
\begin{aligned}
g_{1} \# g_{2} & =g_{1}+g_{2} & & \text { if } g_{1} g_{2} \geqslant 0, \\
& =-2 g_{1}+g_{2} & & \text { if } g_{1}>0, g_{2}<0 \\
& =g_{1}-2 g_{2} & & \text { if } g_{1}<0, g_{2}>0
\end{aligned}
$$

Proposition 2.1. Applying any of the operations R0 to R8 listed below to a plumbing graph $\Gamma$ does not change the oriented diffeomorphism type of $M(\Gamma)$. The same holds for the inverse operations.

R0. (a) If $i$ is a vertex with $g_{i} \geqslant 0$, reverse the signs on all edges other than loops at this vertex.

(b) If $i$ is a vertex with $g_{i}<0$, reverse the sign on any edge at this vertex.

In view of $\mathrm{R} 0$, if $\Gamma^{*}$ is the subgraph of $\Gamma$ defined by all vertices with $g_{i} \geqslant 0$, then the signs on edges are only relevant on cycles of $\Gamma^{*}$. We shall therefore often omit the edge signs of $\Gamma$ which are not on cycles of $\Gamma^{*}$. In fact the information contained in the edge signs of $\Gamma$ is completely coded by the homeomorphism $\varepsilon_{\Gamma}: H_{1}\left(\Gamma^{*}\right) \rightarrow$ $\mathrm{Z} / 2$ which assigns to any cycle of $\Gamma^{*}$ the number modulo 2 of (-)-edges on that cycle. Later we shall work with $\varepsilon_{\Gamma}$, but for the moment edge-signs are more convenient.

R1 (blowing down). In any of the following four situations, replace the graph on the left by the one on the right. Here $\varepsilon= \pm 1$, and $\varepsilon_{0}, \varepsilon_{1}$ and $\varepsilon_{2}$ are edge signs and are related by $\varepsilon_{0}=-\varepsilon \varepsilon_{1} \varepsilon_{2}$.

$$
\Gamma_{0}+\stackrel{\varepsilon}{\bullet} \longrightarrow \Gamma_{0}
$$
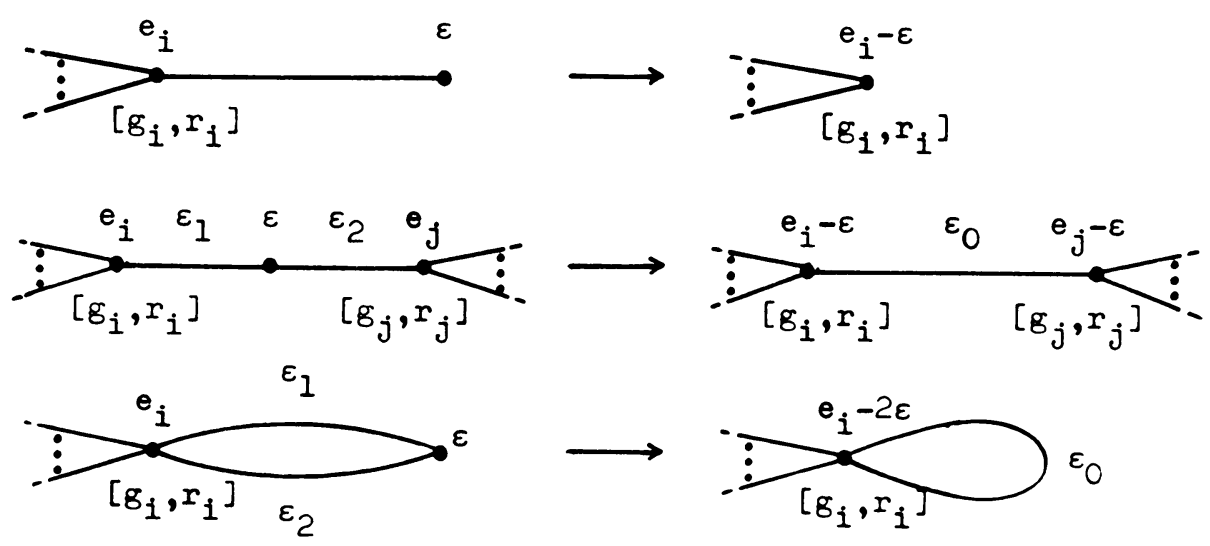
Recall that omitted $g_{i}$ and $r_{i}$ weights are by definition zero. The first case above means any isolated vertex of $\Gamma$ with weights $e= \pm 1, g=0, r=0$ can be deleted.

$\mathrm{R} 2\left(\mathbf{R} P^{2}\right.$-absorption). Here $\delta_{1}= \pm 1, \delta_{2}= \pm 1$, and $\delta=\left(\delta_{1}+\delta_{2}\right) / 2$.
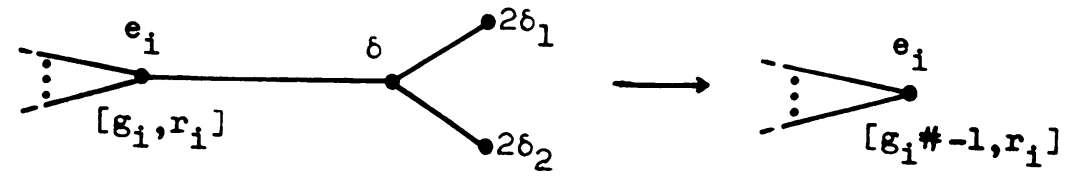

By blowing up (the inverse operation to R1) and blowing down one sees that the three cases of $\mathrm{R} 2$ are mutually equivalent. Indeed

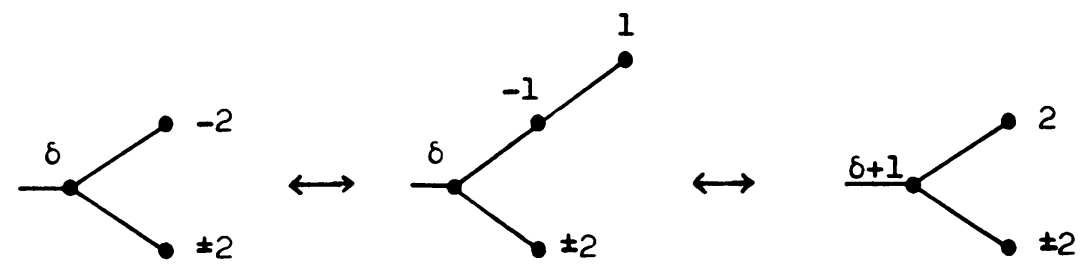

$\mathrm{R} 3$ (0-chain absorption). Here the edge-signs $\varepsilon_{i}^{\prime}$ are given by $\varepsilon_{i}^{\prime}=-\varepsilon \bar{\varepsilon} \varepsilon_{i}$ if the edge in question is not a loop, and $\varepsilon_{i}^{\prime}=\varepsilon_{i}$ if it is a loop.

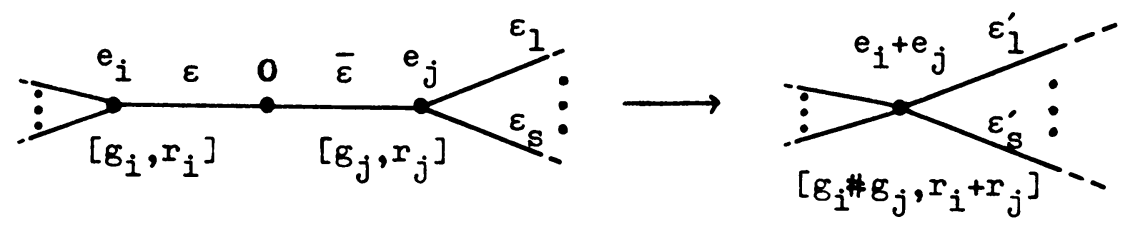

R4 (unoriented handle absorption).
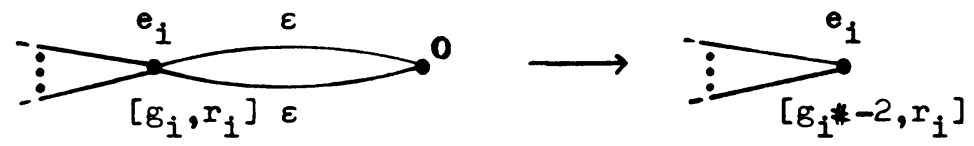

R5 (oriented handle absorption).
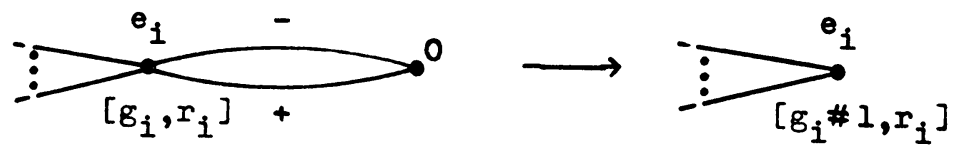

R6 (splitting). If any component of $\Gamma$ has the form

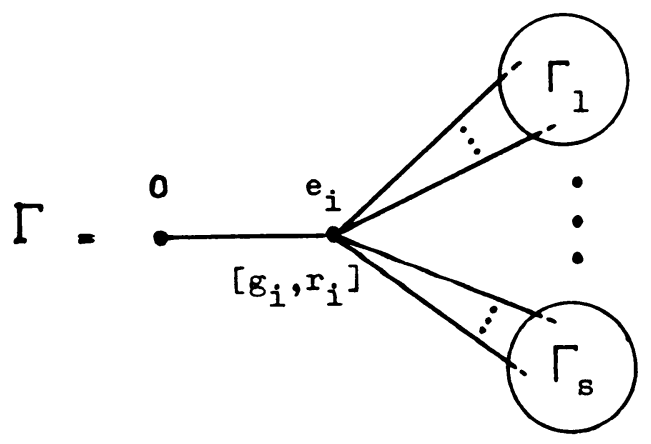


where each $\Gamma_{j}$ is connected and, for each $j, \Gamma_{j}$ is joined to vertex $i$ by $k_{j}$ edges, replace $\Gamma_{0}$ by the disjoint union of $\Gamma_{1}, \ldots, \Gamma_{s}$, and $k$ copies of

$$
0
$$

and $r_{i}$ copies of

$$
[0,1]
$$

where

$$
\begin{aligned}
k & =2 g+\sum_{j=1}^{s}\left(k_{j}-1\right), & & g \geqslant 0 . \\
& =-g+\sum_{j=1}^{s}\left(k_{j}-1\right), & & g<0 .
\end{aligned}
$$

R7 (Seifert graph exchange). If any component of $\Gamma$ is one of the graphs on the left of the following list, replace it by the corresponding graph on the right.

$-1$

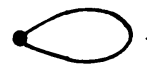

0

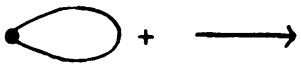

1

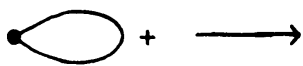

$-1$

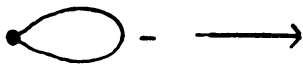

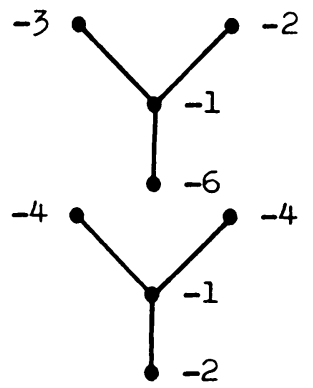

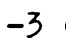

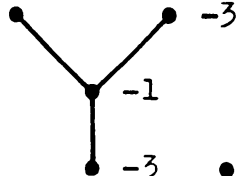

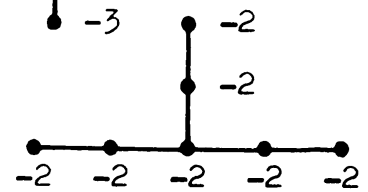

O<smiles>[CH]1CCCCC1</smiles>

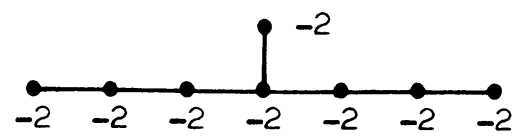

1<smiles>C1CCCCC1</smiles>

R8 (annulus absorption).
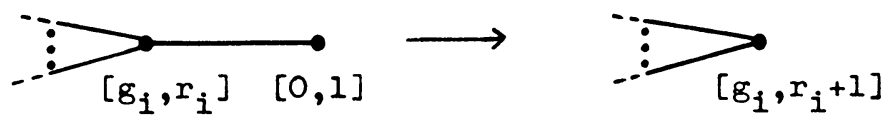
The inverse moves to $\mathrm{R} 1-\mathrm{R} 8$ will be called: blowing up, $\mathbf{R} P^{2}$-extrusion, 0 -chain extrusion, unoriented handle extrusion, oriented handle extrusion, connecting, cyclic graph exchange, annulus extrusion.

Most of the proof of 2.1 is already in the literature, so we just sketch it. RO(a) arises by reversing base and fiber orientations of the bundle $E_{i}$ being plumbed. $\mathrm{R} 0$ (b) arises because if $E_{i}$ has nonorientable base $F_{i}$, then the boundary components of $F_{i}$ do not carry any natural orientation. $\mathrm{R} 1$ is proved in special cases by Von Randow [22]; see also Scharf [21] and Neumann and Weintraub [17]. The general case can be proved the same way; for instance, it is an easy consequence of Lemma 5.4 below. Alternatively, note that once R2, R4, R5, and R8 are proved, by extruding handles and $\mathbf{R} P^{2}$ 's and annuli, $\mathrm{R} 1$ can be reduced to the case $g_{i}=g_{j}=$ $r_{i}=r_{j}=0$ already in the literature. Similar remarks apply to R3 and R6. In fact $\mathrm{R} 3$ is a trivial computation, as are also R5, R6 and R8, so only R2 and R7 need discussion.

For R2 we observe that the manifold determined by the graph

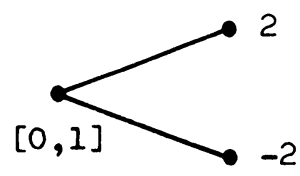

can also be described as the total space $X$ of the $S^{1}$-bundle with orientable total space over the Möbius band $M b[24, \S 3]$. A quick way of seeing this is to think of $X$ as the unit tangent bundle of $M b$. The standard circle action on $M b$ thus lifts to $X$, giving $X$ a Seifert fibration with two 2-fold exceptional fibers. This Seifert manifold structure leads in a standard way (see [7], [16], [22], or $\$ 5$ below) to a plumbing graph, which is easily checked to be the above one. Thus R2 (for $\delta_{1}=1, \delta_{2}=-1$ ) is clear once one verifies that the pasting map is correct, which can also be seen from the above description. As pointed out earlier, the other cases of $R 2$ reduce to this case by blowing up and blowing down.

Before we prove $\mathrm{R} 7$ we need some notation. If $A \in \mathrm{SL}(2, \mathrm{Z})$, let $T(A)$ be the $T^{2}$-bundle over $S^{1}$ with monodromy $A$. That is, $T(A)$ is obtained from $[0,1] \times T^{2}$ by identifying the two ends via the map $A:\{1\} \times T^{2} \rightarrow\{0\} \times T^{2}$. Now the graphs on the left in R7 are all special cases of the cyclic plumbing graphs for torus bundles over $S^{1}$, which we discuss in $\$ 6$, and by the computation of that section, they lead to the torus bundles $T(A)$ with

$$
\begin{aligned}
& A=\left(\begin{array}{ll}
+1 & 1 \\
-1 & 0
\end{array}\right), \quad\left(\begin{array}{cc}
0 & 1 \\
-1 & 0
\end{array}\right), \quad\left(\begin{array}{ll}
-1 & 1 \\
-1 & 0
\end{array}\right), \\
& \left(\begin{array}{cc}
-1 & -1 \\
1 & 0
\end{array}\right), \quad\left(\begin{array}{cc}
0 & -1 \\
1 & 0
\end{array}\right), \quad\left(\begin{array}{cc}
+1 & -1 \\
1 & 0
\end{array}\right)
\end{aligned}
$$

respectively. These $A$ are of finite order 6, 4, 3, 3, 4, 6 respectively, so the corresponding $T(A)$ is always a Seifert manifold. The corresponding Seifert invariants can easily be computed in each case (see for instance [18]; note that the $i$ th example in the list is always the $(7-i)$ th example with reversed orientation, so one need only compute three cases). The result is the following list (using unnormalized Seifert invariants, as in [13] or [16]). 


$$
\begin{aligned}
& (0 ;(1,1),(2,-1),(3,-1),(6,-1)), \\
& (0 ;(1,1),(2,-1),(4,-1),(4,-1)), \\
& (0 ;(1,1),(3,-1),(3,-1),(3,-1)), \\
& (0 ;(1,-1),(3,1),(3,1),(3,1)), \\
& (0 ;(1,-1),(2,1),(4,1),(4,1)), \\
& (0 ;(1,-1),(2,1),(3,1),(6,1)) .
\end{aligned}
$$

But by the standard description of Seifert manifolds by plumbing (due to von Randow [22], but with other orientation conventions; for a description in terms of unnormalized Seifert invariants see [16]) the plumbing graphs on the right in R7 also give this list of Seifert manifolds.

There is of course a great danger of orientation conventions being incompatible somewhere in this proof. If they were so, they would lead to a consistent orientation error in all six examples. We thus need an "orientation safe" method to check at least one of the examples. Such a method is provided by Kirby's link calculus [11]. In general the manifold $M(\Gamma)$ with $\Gamma$ the cyclic graph

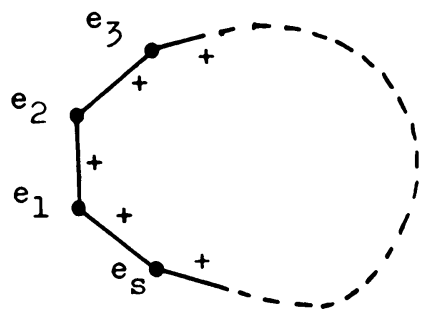

can also be given by surgery on the framed link

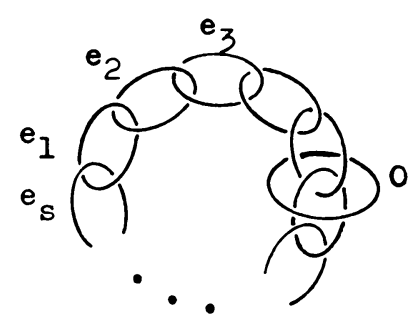

In particular, the first graph on the left in R7, after blowing up once, becomes

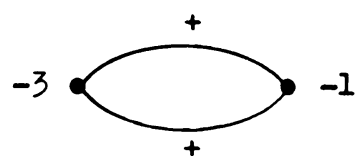

giving the framed link

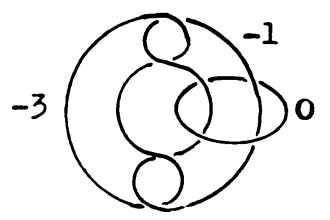


Similarly the graph

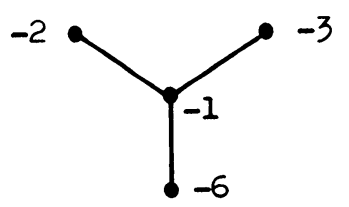

corresponds to the framed link

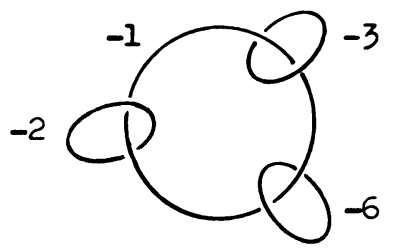

It is now an easy exercise in the link calculus to see both these links can be reduced to the 0 -framed trefoil

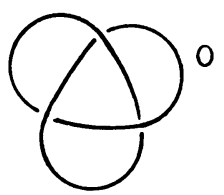

verifying this case. The other cases could of course be verified by the same method, but except for the sixth one this seems less easy.

Warning. The reason we first blew up the first graph is that the framed link corresponding directly to the graph

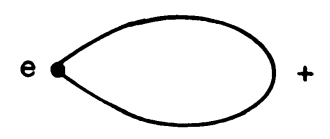

is not, as one might at first expect,

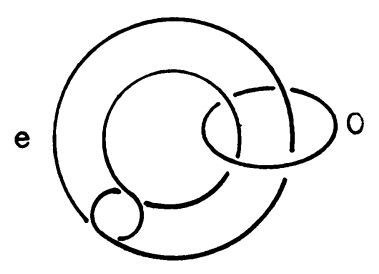

but is

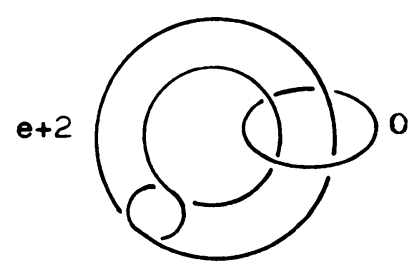


The reason should be clear. With this special case in mind, it is now clear how one can translate any closed plumbing graph $\Gamma$ with all $g_{i}=0$ into a link diagram. (-)-edges in $\Gamma$ translate to linking of the following type:

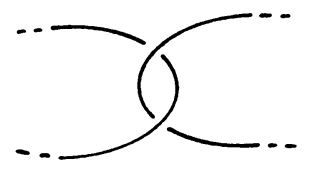

By first doing handle and $\mathbf{R} P^{2}$-extrusions, any closed plumbing graph can be reduced to a graph of this type and then translated into a link diagram. This gives an alternate approach to proving 2.1 , at least in the closed case, and was the approach used in [17].

\section{Sufficiency of the calculus.}

THEOREM 3.1. If $\Gamma_{1}$ and $\Gamma_{2}$ are two plumbing graphs and $M\left(\Gamma_{1}\right) \cong M\left(\Gamma_{2}\right)$, then $\Gamma_{1}$ and $\Gamma_{2}$ are related by operations $\mathrm{R} 0-\mathrm{R} 8$ and their inverses.

To apply this theorem in practice it is usually easiest to use the first step of its proof, namely the reduction to normal form described in $\S 4$. Before describing this we give some corollaries of the theorem and its proof, which give improved versions of the calculus for special classes of graphs.

THEOREM 3.2. For each of the following classes of plumbing graphs Theorem 3.1 holds using just the operations listed and their inverses, and staying within the given class of graphs (undefined operations are defined below).

(i) Orientable plumbing graphs (all $g_{i} \geqslant 0$ ): R0(a), R0(b)', R1, R3, R5, R6, R7, $\mathrm{R} 8, \mathrm{R} 2 / 4$.

(ii) Spherical plumbing graphs (all $g_{i}=0$ ): Same as (i), but omit R5.

(iii) Orientable plumbing graphs with no cycles, so edge signs are irrelevant and can be omitted: R1, R3, R6, R8.

(iv) Star-shaped plumbing graphs for Seifert manifolds: R1.

(v) In Theorem 3.1 and each of the above cases, if only closed plumbing graphs are considered, then $\mathrm{R} 8$ can be omitted.

The undefined operations above are:

R0(b)'. At a vertex where $\mathbf{R} P^{2}$-absorption would be applicable, change the sign of any incident edge.

R2/4. An unoriented handle absorption followed by two $\mathbf{R} P^{2}$-extrusions.

The classes of graphs described in (i) and (ii) are natural classes of plumbing graphs which still give all possible graph-manifolds. The third class is definitely restrictive, but still includes all graph-manifolds which are rational homology spheres. An application of this is given in [15]. The fourth case of the theorem was stated without proof in [16]. As claimed there, it can easily be seen directly.

The following trivial proposition complements the calculus. If $\Gamma$ is a plumbing graph, let $-\Gamma$ be the same graph with the signs of all euler number weights and all edges reversed. 
Proposition 3.3. $-M(\Gamma) \cong M(-\Gamma)$.

\section{Normal form.}

Definition. Two plumbing graphs $\Gamma_{1}$ and $\Gamma_{2}$ are isomorphic, written $\Gamma_{1} \cong \Gamma_{2}$, if there exists a graph isomorphism $\Gamma_{1} \rightarrow \Gamma_{2}$ which respects vertex weights and such that the induced diagram

$$
\begin{array}{ccc}
H_{1}\left(\Gamma_{1}^{*}\right) & \rightarrow & H_{1}\left(\Gamma_{2}^{*}\right) \\
\varepsilon_{\Gamma_{1}} \searrow & & \swarrow \varepsilon_{\Gamma_{2}} \\
& \mathrm{Z} / 2 &
\end{array}
$$

commutes, where $\varepsilon_{\Gamma_{i}}$ is the homomorphism defined just after R0 in $\$ 2$.

Definition. A chain of length $k$ in a plumbing graph $\Gamma$ is any portion of $\Gamma$ of the form (after reindexing vertices)
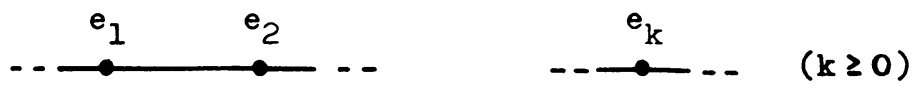

or
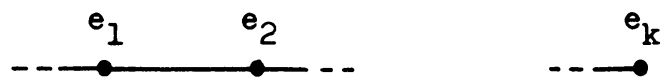

Here the only edges incident to vertices $1, \ldots, k$ are those shown and these edges have arbitrary signs. Recall that by convention our notation indicates that $g_{i}=r_{i}$ $=0$ for $i=1, \ldots, k$. The chain is maximal if it can be included in no larger chain.

The following definition of normal form is based on the following three principles.

(i) Euler number weights should be negative wherever possible.

(ii) Seifert manifolds over orientable surfaces should have their "natural" starshaped plumbing graphs.

(iii) Plumbing graphs should be as small as possible, subject to (i) and (ii).

This preamble hopefully makes the following definition less horrifying.

DEFINITION. $\Gamma$ is in normal form if the following six conditions hold.

N1. None of operations $\mathrm{R} 1-\mathrm{R} 8$ can be applied to $\Gamma$, except that $\Gamma$ may have components of the form

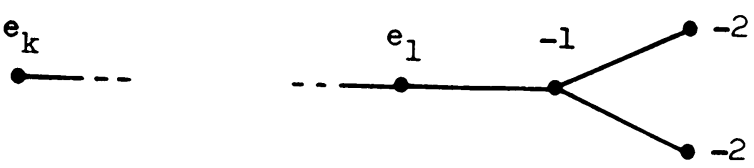

with $k \geqslant 1$ and $e_{i} \leqslant-2$ for $i=1, \ldots, k$.

N2. The weights $e_{i}$ on all chains of $\Gamma$ satisfy $e_{i} \leqslant-2$ (this condition includes much of $\mathrm{Nl}$ ).

N3. No portion of $\Gamma$ has the form

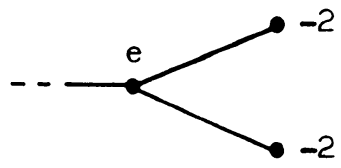


unless it is in a component of $\Gamma$ of the form
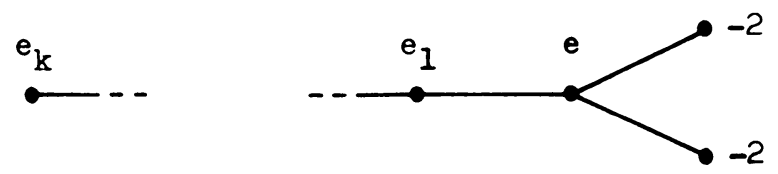

with $k \geqslant 1$ and $e_{i} \leqslant-2$ for $i=1, \ldots, k$.

N4. No portion of $\Gamma$ has the form

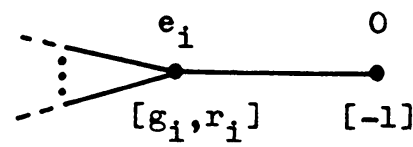

unless vertex $i$ is an interior vertex of a chain.

N5. No component of $\Gamma$ has the form
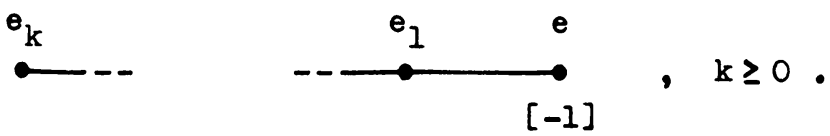

N6. No component of $\Gamma$ is isomorphic to one of the following graphs:
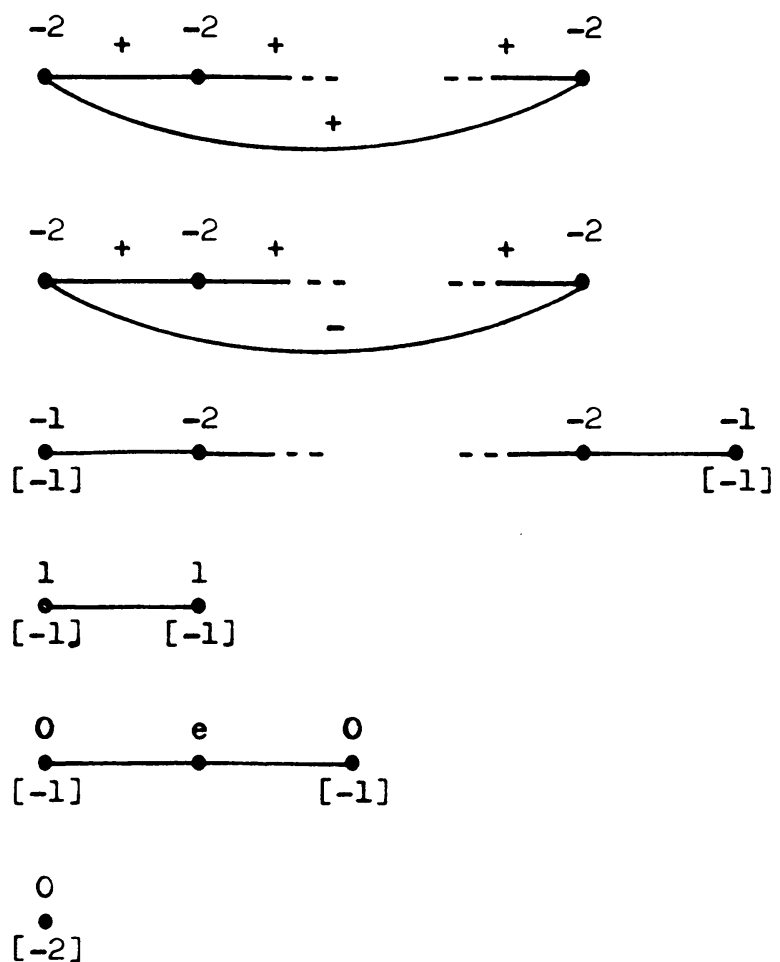

$[-1,1]$ 
THEOREM 4.1. Any plumbing graph can be reduced to normal form using only operations R1-R8 and their inverses.

Proof. We give an algorithm to do what is required. After step $i$ conditions N1 to $\mathrm{N} i$ are satisfied.

Step 1. Apply operations N1-N8 to $\Gamma$ until no more are applicable. This process clearly terminates.

Step 2a. Suppose we have a maximal chain
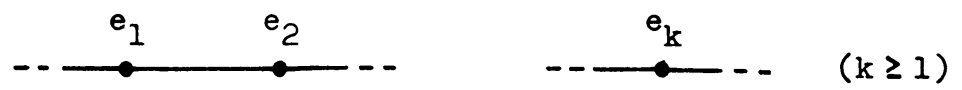

embedded in $\Gamma$ as follows:
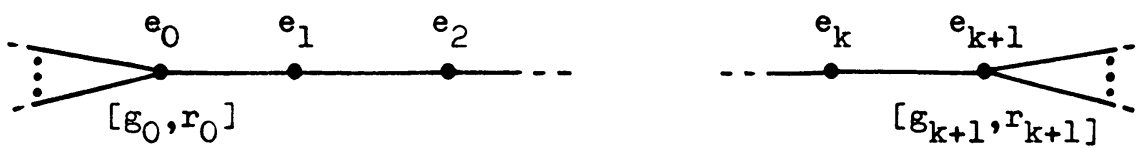

where we are omitting the edge signs for convenience. Here the vertices labelled 0 and $k+1$ may coincide; however we assume for the moment that the chain is not part of a cyclic component

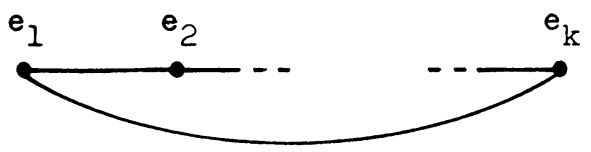

of $\Gamma$. Because of step 1 we know $\left|e_{i}\right| \geqslant 2$ for $i=1, \ldots, k$. We convert this chain into one, all of whose weights are $\leqslant-2$, as follows.

Let $i \leqslant k$ be the largest index with $e_{i} \geqslant 2$. By doing $\left(e_{i}-1\right)(-1)$-blow-ups directly to the left of this vertex we obtain the following situation (the effect on the omitted edge signs is understood):

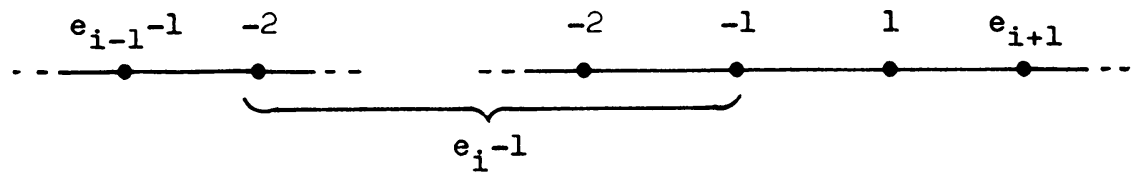

(with the obvious interpretation of what this means if $i=1$ or $k$ ). Now blow down the 1-weighted vertex to get

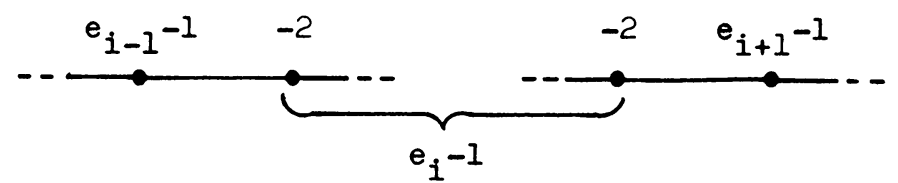

If $e_{i-1} \neq 2$ we now have a chain like the one we started with, but with less positive weights. If $e_{i-1}=\cdots=e_{i-j}=2$ this will be true after doing $j$ blow-downs to get
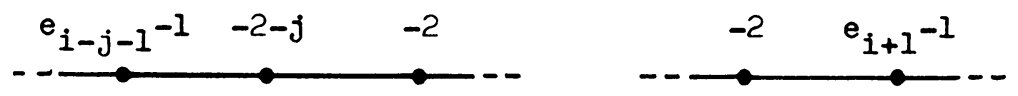
In any case we now repeat the process until eventually our chain has the desired form.

The same procedure can be applied to a chain of the type
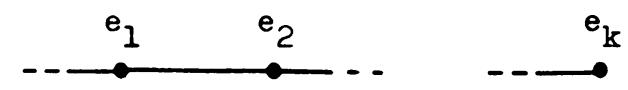

Step 2b. To achieve condition N2 of the definition of normal form it only remains to describe how to deal with cyclic components

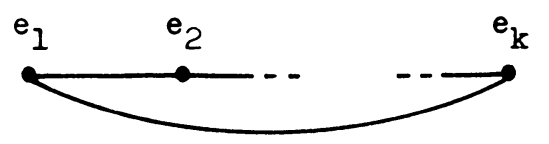

of $\Gamma$. The procedure of step $2 \mathrm{a}$ still works in this case to produce a cycle with all weights $\leqslant-2$ except possibly when $e_{1}=2$. Since we can cyclically permute the indices, the only case we must deal with is therefore the case that $e_{1}=e_{2}=\cdots=$ $e_{k}=2$. In this case the procedure of step 2 results in the graph

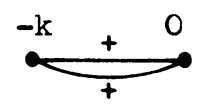

or

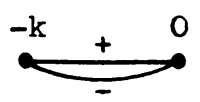

and we then apply a handle absorption (R4 or R5) to reduce this to

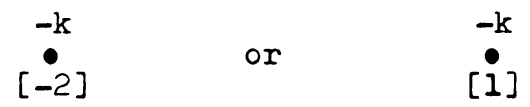

respectively.

Step 2c. Step 2a may have made additional $\mathbf{R} P^{2}$-absorptions possible. If so, do them.

Step 3. Given a portion of $\Gamma$ as in condition N3, apply the following operation, followed by the procedure of step 2 if $e \geqslant-2$.

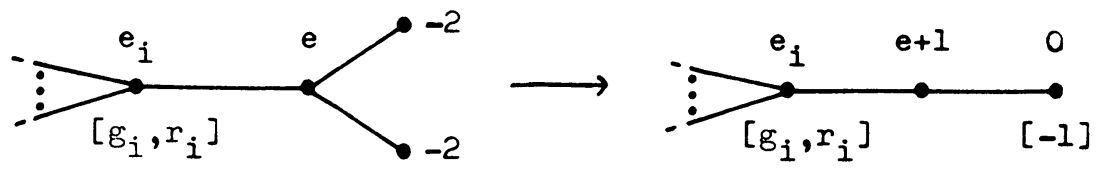

This is a 0 -chain extrusion to get

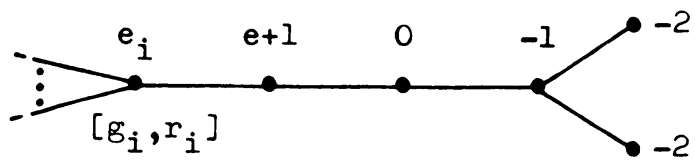

followed by an $\mathbf{R} P^{2}$-absorption. 
Step 4. Given a portion of $\Gamma$ as in N4, apply the following operation (an $\mathbf{R} P^{2}$-extrusion followed by a 0 -chain absorption).

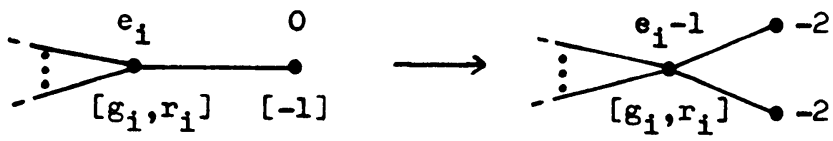

Step 5. Replace any component of $\Gamma$ isomorphic to a graph on the left below by the corresponding graph on the right.
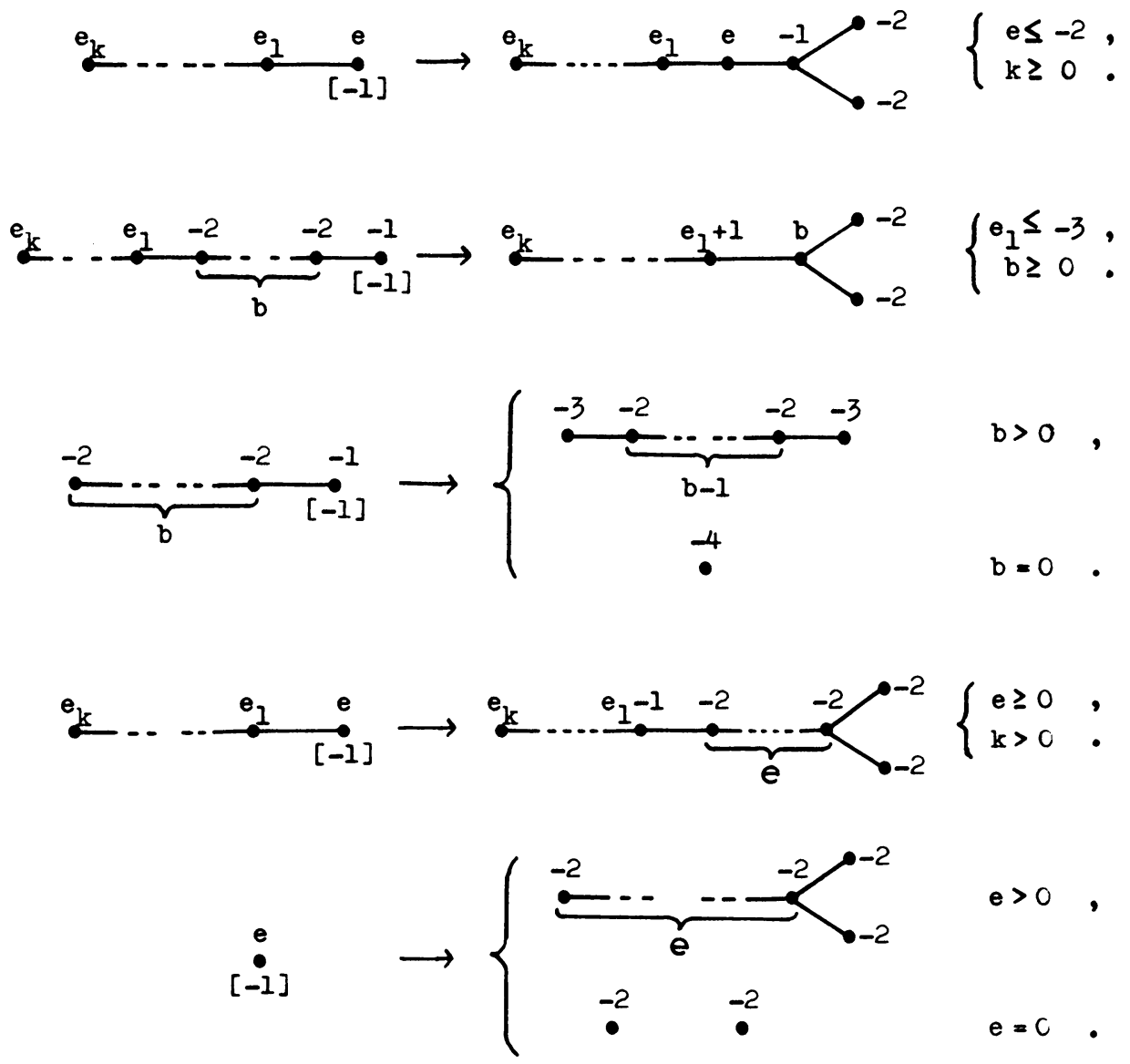

Each of these is an $\mathbf{R} P^{2}$-extrusion followed by blow-downs and the procedure of step $2 \mathrm{a}$ if necessary, except that the final case involves a splitting operation instead.

Step 6. Replace any component of $\Gamma$ isomorphic to a graph on the left below by the corresponding graph on the right. 

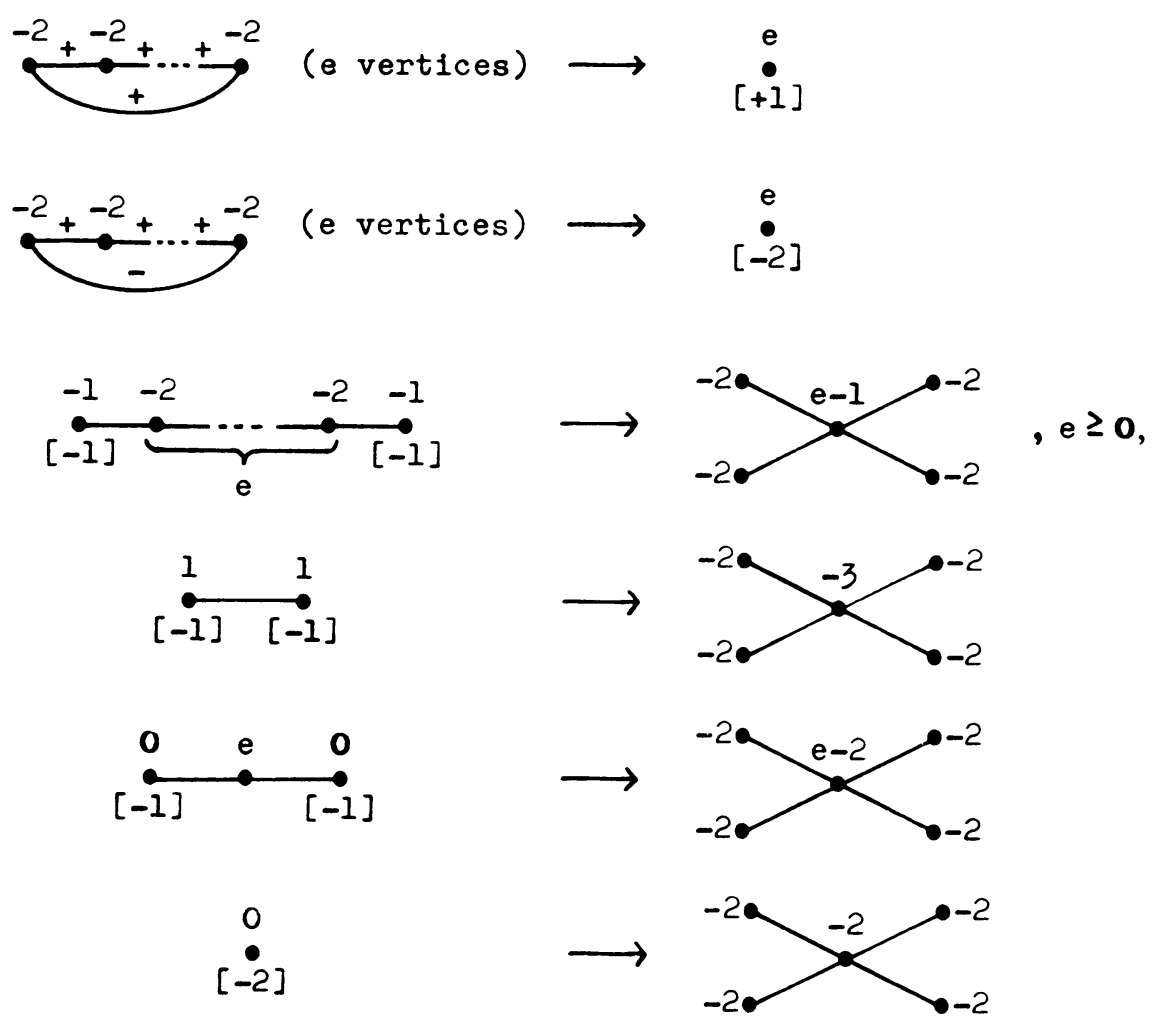

$$
[-1,1]
$$

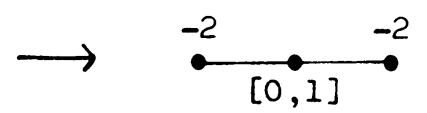

The first two of these are most easily explained from right to left; they are then a handle extrusion followed by the procedure of step 2 . The third to sixth are two $\mathbf{R} P^{2}$-extrusions followed by blow-downs and 0-chain absorptions where necessary. The last is an $\mathbf{R} P^{2}$-extrusion followed by an annulus absorption.

This completes the proof of Theorem 4.1. The next theorem completes the description of the calculus.

THEOREM 4.2. If $M\left(\Gamma_{1}\right) \cong M\left(\Gamma_{2}\right)$ and $\Gamma_{1}$ and $\Gamma_{2}$ are in normal form then $\Gamma_{1} \cong \Gamma_{2}$.

In view of uniqueness of prime decomposition for 3-manifolds the following theorem reduces everything to the case of connected plumbing graphs.

THEOREM 4.3. If $\Gamma$ is a connected normal form plumbing graph then $M(\Gamma)$ is prime (that is, it cannot be written as a nontrivial connected sum).

We shall prove the above two theorems in the next two sections. The following is a useful corollary of Theorem 4.3. 
COROLlaRY 4.4. If $\Gamma$ is a connected graph which admits no blowing down, no 0 -chain absorption and no splitting, then either $M(\Gamma)$ is prime or

$$
\Gamma=\frac{0}{[-1]}
$$

and $M(\Gamma) \cong \mathbf{R} P^{3} \# \mathbf{R} P^{3}$.

PRoof. If we use the above algorithm to reduce $\Gamma$ to normal form, the only way $\Gamma$ can become disconnected is via the last case of step 5 , if

$$
\Gamma=\stackrel{0}{\cdot}\left[\begin{array}{c}
-1] \\
{[-1}
\end{array}\right.
$$

Theorem 3.1 is an immediate corollary of Theorems 4.1 and 4.2. Theorem 3.2 can be proved similarly by giving a suitable normal form for graphs in each of the four special classes described there. An inelegant but adequate method is as follows. Define a graph $\Gamma$ to be in 0-normal form if it results from a graph in normal form by doing $\left|g_{i}\right| \mathbf{R} P^{2}$-extrusions at each vertex with $g_{i}<0$. $\Gamma$ is in $S$-normal form if it results from a graph in 0-normal form by doing $g_{i}$ oriented handle extrusions at each vertex with $g_{i}>0$. Clearly a graph in normal form determines a unique graph in 0 -normal form or $S$-normal form and vice versa. Thus Theorem 4.2 applies also to these normal forms. Now 0-normal form is suitable for classes (i), (iii) and (iv) of Theorem 3.2 and $S$-normal form is suitable for (ii) (and also (i)). Thus to prove 3.2 it suffices to show that for each case one can reduce within the relevant class to the relevant normal form using the given operations. This easy verification is left to the reader.

5. Reduced graphs. We shall prove Theorems 4.2 and 4.3 simultaneously, using Waldhausen's classification [24] of graph manifolds. We thus assume throughout this section: $\Gamma$ is a connected plumbing graph in normal form.

There are two cases that do not fit conveniently into Waldhausen's classification and will be discussed in detail in the next section. These are the cases of a straight line graph or a cyclic graph, that is a graph of the form

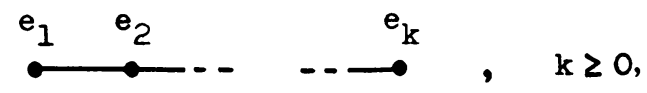

or

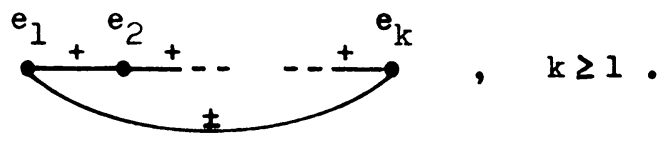

We will thus exclude these cases much of the time in this section.

Now recall that each edge $\sigma$ of $\Gamma$ corresponds to an embedded torus $T_{\sigma} \subset M(\Gamma)$ and that the collection of all these tori cuts $M(\Gamma)$ open into a disjoint union of circle bundles over compact surfaces. Such a collection of tori is called a graph 
structure on $M(\Gamma)$ by Waldhausen. We want a subcollection which is still a graph structure and which is reduced in Waldhausen's sense, which we explain later.

THEOREM 5.1. Assume $\Gamma$ is a connected normal form plumbing graph which is not a straight line graph. Let $S$ be a subset of the set of edges of $\Gamma$ which contains precisely one edge of each maximal chain of $\Gamma$. Then $\left\{T_{\sigma} \mid \sigma \in S\right\}$ is a reduced graph structure on $M(\Gamma)$.

We shall define reduced graph structure once we are ready to prove the theorem, but first we need some preparatory notation and computations.

If $b_{1}, \ldots, b_{k}$ are real numbers, we denote

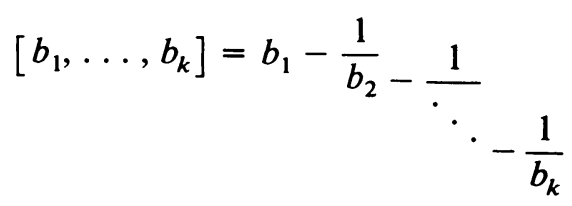

and

$$
A\left(b_{1}, \ldots, b_{k}\right)=\left(\begin{array}{cc}
b_{k} & 1 \\
-1 & 0
\end{array}\right) \cdots\left(\begin{array}{cc}
b_{1} & 1 \\
-1 & 0
\end{array}\right) .
$$

For $k=0$ we also define $A()=\left(\begin{array}{ll}1 & 9 \\ 0 & 1\end{array}\right)=J$.

Lemma 5.2. Define recursively $P_{-1}=0, P_{0}=1, P_{i+1}=b_{i+1} P_{i}-P_{i-1} ; Q_{-1}=$ $-1, Q_{0}=0, Q_{i+1}=b_{i+1} Q_{i}-Q_{i-1}$.

(i) $\left[b_{1}, \ldots, b_{k}\right]=P_{k} / Q_{k}$ and

$$
A\left(b_{1}, \ldots, b_{k}\right)=\left(\begin{array}{cc}
P_{k} & Q_{k} \\
-P_{k-1} & -Q_{k-1}
\end{array}\right) .
$$

(ii) If $b_{i} \geqslant 2$ for all $i$ then $P_{i+1} \geqslant P_{i}+1$ and $Q_{i+1} \geqslant Q_{i}+1$, so in particular $P_{i} \geqslant i+1$ and $Q_{i} \geqslant i$ for all $i$.

(iii) If moreover some $b_{i}>2$, then $P_{k}>P_{k-1}+1$.

Proof. The proof is a trivial induction.

Returning to Theorem 5.1, we first show that the set of tori in question is a graph structure for $M(\Gamma)$.

Suppose we have (after suitably reindexing vertices) a maximal chain of length $k$ embedded in $\Gamma$ as follows:
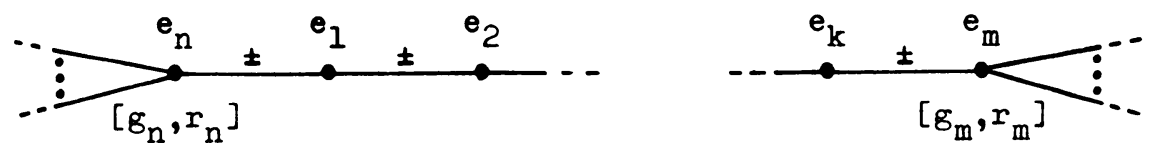

For the moment assume $m \neq n$. The bundle of euler number $e_{i}$ over $S^{2}$ can be pasted together from two trivial bundles over $D^{2}$ as follows:

$$
D^{2} \times S^{1} \cup_{H_{i}} D^{2} \times S^{1}, \quad H_{i}=\left(\begin{array}{cc}
-1 & 0 \\
-e_{i} & 1
\end{array}\right): S^{1} \times S^{1} \rightarrow S^{1} \times S^{1} .
$$


The -1 in the upper left of $H_{i}$ reflects the fact that the base circle $S^{1} \times\{1\}$ inherits opposite orientations from each side. The bundle over the twice punctured $S^{2}$, needed for plumbing, is thus $A \times S^{1} \cup_{H_{i}} A \times S^{1}$ where $A \cong I \times S^{1}$ is an annulus. The plumbing according to the above portion of $\Gamma$ thus gives the pasting (with $\left.J=\left(\begin{array}{ll}0 & 1 \\ 1 & 0\end{array}\right)\right)$ :

$$
E_{n} \cup_{ \pm J}\left(A \times S^{1} \cup_{H_{1}} A \times S^{1}\right) \cup_{ \pm J} \ldots \cup_{ \pm J}\left(A \times S^{1} \cup_{H_{k}} A \times S^{1}\right) \cup_{ \pm J} E_{m} \text {, }
$$

where $E_{n}$ and $E_{m}$ are the bundles corresponding to vertices $n$ and $m$. Since $A \times S^{1} \cong I \times T^{2}$ is a collar, this is equivalent to the pasting

$$
E_{n} \cup_{C} E_{m}, \quad C=(-1)^{c} B, \quad B=J H_{k} J \cdots J H_{1} J,
$$

where $c$ is the number of $(-)$-edges on the chain.

A similar analysis applies if $n=m$. Also similarly a chain
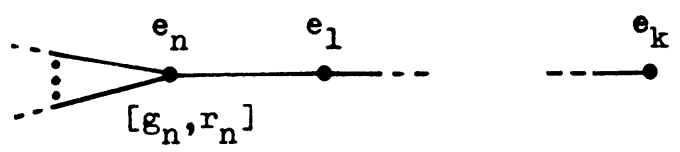

in $\Gamma$ leads to the pasting

$$
E_{n} \cup_{B} S^{1} \times D^{2}, \quad B \text { as in (*) above. }
$$

The initial factor $J$ in $B$ in this case arises because we wrote $E_{n} \cup S^{1} \times D^{2}$ instead of $E_{n} \cup D^{2} \times S^{1}$.

The statement that the set in Theorem 5.1 is a graph structure is now clear. To show it is reduced we need some more computations.

LEMMA 5.3. The matrix $B$ of (*) and (**) above satisfies:

(i) In the terminology of Lemma 5.2 with $b_{i}=-e_{i}$,

$$
B=A\left(b_{1}, \ldots, b_{k}\right) J=\left(\begin{array}{cc}
Q_{k} & P_{k} \\
-Q_{k-1} & -P_{k-1}
\end{array}\right) ;
$$

(ii) if $k \geqslant 0$ then $P_{k} \neq 0$;

(iii) if $k \geqslant 1$ then $P_{k-1} \neq 0$ and $P_{k} \geqslant 2$.

Proof. (i) is immediate, since

$$
J H_{i}=\left(\begin{array}{cc}
b_{i} & 1 \\
-1 & 0
\end{array}\right)
$$

and (ii) and (iii) follow from 5.2(ii) since $b_{i} \geqslant 2$ by definition of normal form.

We shall need two special cases of the above. Let $X$ be the oriented total space of an $S^{1}$-bundle over the Möbius band and parametrize $\partial X=S^{1} \times S^{1}$ so the first factor is the boundary of a section of $X$ and the second factor is a fiber. Consider a chain in $\Gamma$ of the form
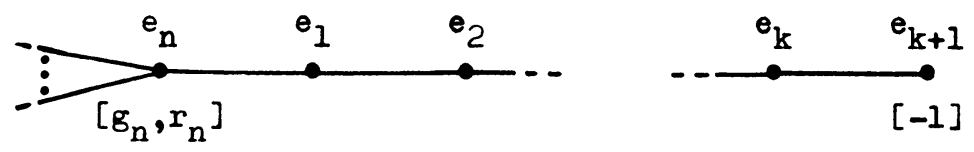
LEMMA 5.4. (i) This chain yields the pasting $E_{n} \cup_{D} X$ with

$$
D=\left(\begin{array}{cc}
0 & 1 \\
-1 & 0
\end{array}\right) A\left(b_{1}, \ldots, b_{k+1}\right) J=\left(\begin{array}{cc}
-Q_{k} & -P_{k} \\
-Q_{k+1} & -P_{k+1}
\end{array}\right),
$$

where $b_{i}=-e_{i}$ for $i=1, \ldots, k+1$.

(ii) In this situation $P_{k+1} \neq 0$.

Proof. For part (i) apply a 0-chain extrusion to get
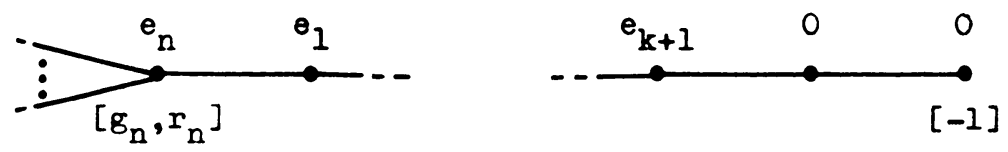

and apply the computation preceding Lemma 5.2. For part (ii) observe that $b_{i} \geqslant 2$ for $i=1, \ldots, k$, by definition of normal form. If $k \geqslant 1$ then $P_{k+1}=b_{k+1} P_{k}-$ $P_{k-1}$, which cannot be zero by Lemma 5.2(ii). If $k=0$ then $P_{k+1}=b_{k+1}=b_{1}$, which is not zero by condition N4 for normal form.

Now suppose $\Gamma$ has the form

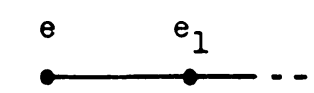

$[-1]$

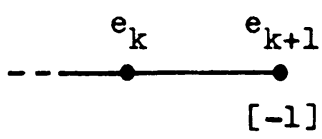

LEMmA 5.5. (i) This $\Gamma$ yields $M(\Gamma)=X \cup_{E} X$ with

$$
E=D\left(\begin{array}{cc}
-1 & 0 \\
-e & -1
\end{array}\right)=\left(\begin{array}{cc}
Q_{k}+e P_{k} & P_{k} \\
Q_{k+1}+e P_{k+1} & P_{k+1}
\end{array}\right)
$$

with $D$ as in the previous lemma.

(ii) In this situation $Q_{k+1}+e P_{k+1} \neq 0$.

Proof. Part (i) is a similar argument to 5.4(i). For part (ii) first note that $P_{k+1}$ and $Q_{k+1}$ are coprime, since det $D=-1$. Assume

$$
Q_{k+1}+e P_{k+1}=0 .
$$

Then since $P_{k+1} \neq 0$ by 5.4(ii), we must have $P_{k+1}= \pm 1$, that is

$$
P_{k+1}=b_{k+1} P_{k}-P_{k-1}= \pm 1 \text {. }
$$

There are now three possibilities.

Case 1. If $k>1$ then 5.2(ii) and (B) imply $b_{1}=\cdots=b_{k}=2$ and $b_{k+1}=1$. This gives $P_{k+1}=Q_{k+1}=1$, so $e=-1$ by (A). $\Gamma$ is thus the third graph excluded by condition N6 for normal form.

Case 2. If $k=1$ then $P_{k}=b_{1} \geqslant 2$ and $P_{k-1}=1$. Thus, by (B), either $b_{2}=0$, or $b_{1}=2$ and $b_{2}=1$. If $b_{2}=0$ then $Q_{k+1}=0$ so (A) gives $e=0$, so $\Gamma$ is the fifth graph excluded by N6. If $b_{1}=2$ and $b_{2}=1$ then as in case 1 we get a special case of the third graph excluded by N6.

Case 3. If $k=0$ then $P_{k+1}=b_{1}$, so $b_{1}= \pm 1$. Since $Q_{k+1}=Q_{1}=1$, (A) gives $e= \pm 1$, so $\Gamma$ is either the fourth graph excluded by N6 or a special case of the third graph excluded by N6. 
Thus none of these can occur, so the proof is complete.

We are now finally ready to define "reduced graph structure" and complete the proof of Theorem 5.1. Let $T$ be the union of the $T_{\sigma}, \sigma \in S$, and let $M_{0}$ be $M(\Gamma)$ cut open along $T$. We think of $M=M(\Gamma)$ as pasted together from the components of $M_{0}$. Thus to any fixed $T_{\sigma}, \sigma \in S$, we have components $M_{1}$ and $M_{2}$ of $M_{0}$, which may coincide, which in $M$ are pasted along $T_{\sigma}$. Waldhausen defines a reduced graph structure to be one in which none of the following ten situations occurs, with indices as written or transposed. For each one we give in parentheses the reason why it does not occur in our case.

W1. $M_{1} \neq M_{2}$ and $M_{1} \cong A \times S^{1}$ where $A$ is the annulus. ( $\Gamma$ admits no annulus absorptions and $S$ contains just one edge of any maximal chain.)

W2. Fiber of $M_{1}$ homologous in $T_{\sigma}$ to a fiber of $M_{2}$ (Lemma 5.2 (ii)).

W3. $M_{1} \cong S^{1} \times D^{2}$ and a meridian $\{p\} \times S^{1} \subset \partial M_{1}=T_{\sigma}$ has intersection number \pm 1 in $T_{\sigma}$ with a fiber of $M_{2}\left(P_{k} \geqslant 2\right.$ in 5.3(iii)).

W4. $M_{1} \cong S^{1} \times D^{2}$ and a meridian $\{p\} \times S^{1} \subset \partial M_{1}=T_{\sigma}$ is homologous in $T_{\sigma}$ to a fiber of $M_{2}\left(P_{k-1} \neq 0\right.$ in 5.3(iii)).

W5. $M_{1} \cong X$ (the $S^{1}$-bundle over the Möbius band) and the homology class $\mu_{1}$ in $\partial M_{1}=T_{\sigma}$ of the boundary of a section of $M_{1}$ is homologous to a fiber of $M_{2}$ in $T_{\sigma}$ (Lemma 5.4(ii)).

W6. $M_{1} \cong X$ and $M_{2} \cong X$ and $\mu_{1}$ is homologous to $\mu_{2}$ where $\mu_{i}$ is defined as in W5 (Lemma 5.5(ii)).

W7. Cutting $M$ only along $T_{\sigma}$ splits it into the graph manifold given by

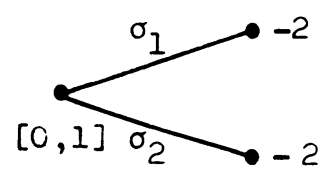

and a piece which is not a solid torus, and $T_{\sigma_{i}}, i=1,2$, are included in the graph structure (condition $\mathrm{N} 3$ in the definition of normal form).

W8. $M_{1}=M_{2} \cong A \times S^{1}=I \times S^{1} \times S^{1}$ and the pasting map $S^{1} \times S^{1} \rightarrow S^{1} \times$ $S^{1}$ is given by a matrix of trace \pm 2 . ( $\Gamma$ would be a cyclic plumbing graph; we show the relevant trace has absolute value $\geqslant 3$ in the next section.)

W9. $M_{1}=D^{2} \times S^{1}$ and $M_{2}=D^{2} \times S^{1}$. ( $\Gamma$ would be a straight line graph, excluded by hypothesis.)

W10. $M=M_{0}$ is an $S^{1}$-bundle over $S^{2}$ or $\mathbf{R} P^{2}$. (A bundle over $S^{2}$ is a special case of a straight line graph; bundles over $\mathbf{R} P^{2}$ are excluded by condition N5 of normal form.)

This completes the proof of Theorem 5.1. Theorem 4.3 now also follows, since by Waldhausen [24] a manifold possessing a reduced graph structure is irreducible, hence prime, while the straight line graphs excluded in Theorem 5.1 yield lens spaces which are also prime.

REMARK. W9 and W10 above are slightly unfortunate. Suppose one replaces them by:

W9'. $M_{1}=D^{2} \times S^{1}$ and $M_{2}=D^{2} \times S^{1}$ and $M$ can also be expressed as a circle bundle over $S^{2}$. 
One obtains a perfectly good modified definition of reduced graph structure, and straight line graphs and lens spaces no longer play a special role anywhere in this section. We will follow Waldhausen's convention however.

Following Waldhausen, we call a manifold with a chosen reduced graph structure a reduced graph manifold. To any reduced graph manifold $M_{1}$ other than a torus bundle over $S^{1}$ with graph structure consisting of a single fiber (which we call the canonical graph structure on a torus bundle), Waldhausen associates a weighted graph $G$, which completely codes the topology of the reduced graph manifold. We shall compute this graph $G$, which we call the $W$-graph corresponding to $\Gamma$, for the graph structure on $M(\Gamma)$ of Theorem 5.1.

We must first describe Waldhausen's graphs. Thus let $M$ be a reduced graph manifold as above and let $\left\{T_{\sigma}, \sigma \in S\right\}$ be its reduced graph structure. Let $M_{0}$ be $M$ cut open along all the $T_{\sigma}$.

The $W$-graph $G$ has one vertex $i$ to each component $M_{i}$ of $M_{0}$ and one edge $\sigma$ for each $T_{\sigma}$ of the graph structure, this edge connecting the vertices $i$ and $j$ corresponding to the components $M_{i}$ and $M_{j}$ of $M_{0}$ which glue along $T_{\sigma}$ ( $i$ and $j$ may coincide). Furthermore $G$ has the following additional structure:

(i) Each vertex $i$ with $M_{i} ¥ S^{1} \times D^{2}$ carries a weight $\left(g_{i}, r_{i}, s_{i}\right)$, where $g_{i}$ and $r_{i}$ have the same meaning (genus and number of "free" boundary components) as in $\Gamma$, and $s_{i}$ is a cross-section obstruction, which we describe below, which vanishes if $r_{i} \neq 0$. Vertices with $M_{i} \cong S^{1} \times D^{2}$ are left unweighted.

(ii) Each edge $\sigma$ is arbitrarily assigned a direction. Moreover this edge $\sigma$ carries a weight $\left(\alpha_{\sigma}, \beta_{\sigma}\right)$ satisfying

$$
\left\{\begin{array}{l}
\operatorname{gcd}\left(\alpha_{\sigma}, \beta_{\sigma}\right)=1, \quad 0 \leqslant \beta_{\sigma}<\alpha_{\sigma} \\
0<\beta_{\sigma}, \quad \text { if } \sigma \text { is incident on an unweighted vertex. }
\end{array}\right.
$$

(Waldhausen requires an edge incident on an unweighted vertex to be directed toward this vertex. This is unnecessary, so we drop this requirement.)

(iii) If $G^{*}$ is the full subgraph of $G$ on the weighted vertices with $g_{i} \geqslant 0$, then a homomorphism $\varepsilon_{G}: H_{1}\left(G^{*}\right) \rightarrow \mathbf{Z} / 2$ is given.

We first describe the weights $\left(\alpha_{\sigma}, \beta_{\sigma}\right)$. First, for each vertex $i$ we choose fiber orientations in each boundary component of $M_{i}$ such that these fiber orientations are mutually compatible if $M_{i}$ has orientable base. If $\sigma$ is an edge directed from vertex $i$ to vertex $j$, let $\left\{\mu_{\sigma}^{+}, \nu_{\sigma}^{+}\right\}$be the following basis of $H_{1}\left(T_{\sigma}\right)$. If $M_{j} \approx S^{1} \times$ $D^{2}$, choose $\mu_{\sigma}^{+}$as the class of a fiber of $M_{j}$. If $M_{j} \simeq S^{1} \times D^{2}$, choose $\mu_{\sigma}^{+}$as the class of a meridian $\{1\} \times S^{1}$ of $S^{1} \times D^{2}$. We shall choose $\nu_{\sigma}^{+}$so that $\nu_{\sigma}^{+}, \mu_{\sigma}^{+}$form an oriented basis of $H_{1}\left(T_{\sigma}\right)$ with $T_{\sigma}$ oriented as part of $\partial M_{j}$. Thus $\nu_{\sigma}^{+}$is so far only determined up to adding multiples of $\mu_{\sigma}^{+}$. We define a basis $\left\{\mu_{\sigma}^{-}, \nu_{\sigma}^{-}\right\}$of $H_{1}\left(T_{\sigma}\right)$ the same way, using $M_{i}$ instead of $M_{j}$.

An easy computation (see [24]) shows that we can choose $\nu_{\sigma}^{+}$and $\nu_{\sigma}^{-}$so that the following relations hold:

$$
\begin{aligned}
\mu_{\sigma}^{+} & =\varepsilon_{\sigma}\left(\alpha_{\sigma} \nu_{\sigma}^{-}+\beta_{\sigma} \mu_{\sigma}^{-}\right), \quad \mu_{\sigma}^{-}=\varepsilon_{\sigma}\left(\alpha_{\sigma} \nu_{\sigma}^{+}+\beta_{\sigma}^{\prime} \mu_{\sigma}^{+}\right) \\
\varepsilon_{\sigma} & = \pm 1, \quad 0 \leqslant \beta_{\sigma}<\alpha_{\sigma}, \quad 0 \leqslant \beta_{\sigma}^{\prime}<\alpha_{\sigma}, \quad \beta_{\sigma} \beta_{\sigma}^{\prime} \cong 1 \bmod \left(\alpha_{\sigma}\right),
\end{aligned}
$$


and $\nu_{\sigma}^{-}$and $\nu_{\sigma}^{+}$are then well defined. The reducedness criteria imply $0<\beta_{\sigma}<\alpha_{\sigma}$ if $i$ or $j$ is an unweighted vertex.

Next the weights $s_{i}$ are given as follows. If $i$ is a vertex with $M_{i} \approx S^{1} \times D^{2}$ and $r_{i}=0$, then the $\nu_{\sigma}^{ \pm}$of adjacent edges define a section on $\partial M_{i}$ and we put $s_{i}$ equal to the cross-section obstruction to extending this section over $M_{i}$.

Finally if $G^{*}$ is the subgraph in (iii) above, define $\varepsilon_{G}: H_{1}\left(G^{*}\right) \rightarrow \mathrm{Z} / 2$ by putting $\varepsilon(C)$ equal to the number modulo 2 of negative $\varepsilon_{\sigma}$ with $\sigma \in C$, for any cycle $C$ of $G^{*}$.

If $\sigma$ is any edge of $G$, we consider the graph obtained by reversing the direction of $\sigma$ and replacing $\beta_{\sigma}$ by $\beta_{\sigma}^{\prime}$ to be equivalent to $G$. As Waldhausen shows, $G$ is then determined (up to equivalence) by, and determines, the reduced graph manifold $M$ (that is $M$ plus its reduced graph structure). Of course the reducedness criteria impose some restrictions on the form of a $W$-graph $G$. We shall not list them, since it is a simple exercise for the reader, and they are also listed by Waldhausen.

Now let $\Gamma$ be a connected normal form plumbing graph which is not a straight line graph or a cyclic graph (as remarked earlier, the exclusion of straight line graphs can be avoided). Let $\left\{T_{\sigma}, \sigma \in S^{1}\right\}$ be the reduced graph structure on $M=M(\Gamma)$ of Theorem 5.1. We describe how to obtain the corresponding $W$-graph $G$.

Step 1. Replace any maximal chain in $\Gamma$,
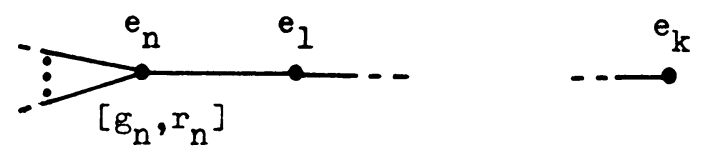

or
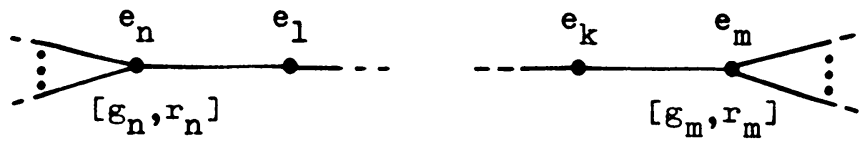

with $k \geqslant 0$ (in the second case $n$ and $m$ may coincide) by a directed weighted edge

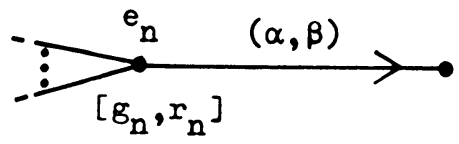

or

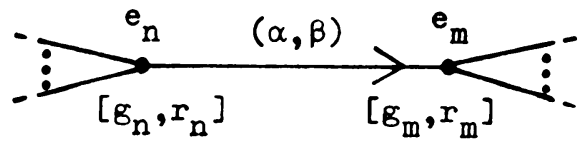

respectively, where $(\alpha, \beta)=(p, p-q)$ and $(p, q)$ is determined by the continued fraction $p / q=\left[b_{1}, \ldots, b_{k}\right], b_{i}=-e_{i}$. 
Step 2. Replace each maximal chain of length 0 in $\Gamma$,

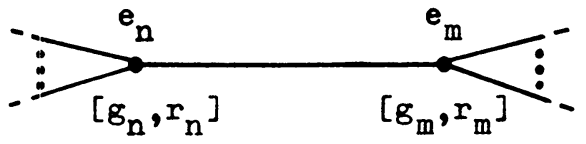

( $n$ and $m$ may coincide) by an oriented weighted edge

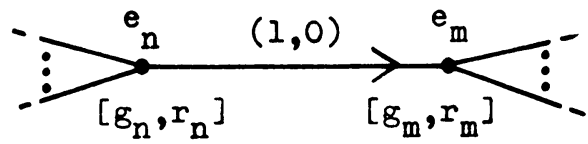

Step 3. We wish to assign weights $\left(g_{i}, s_{i}, r_{i}\right)$ to those vertices which should have them. Only $s_{i}$ needs determining, and that only when $r_{i} \neq 0$. We then put $s_{i}=e_{i}+$ $c_{i}$ where $c_{i}$ is the degree of vertex $i$ in the subgraph $G_{0}$ of $G$ obtained by deleting the edges described in step 2 (that is the $(1,0)$-weighted edges).

Step 4. $\varepsilon_{G}: H_{1}\left(G^{*}\right) \rightarrow \mathbf{Z} / 2$ is the homomorphism induced in the obvious way from $\varepsilon_{\Gamma}: H_{1}\left(\Gamma^{*}\right) \rightarrow \mathbf{Z} / 2$.

THEOREM 5.6. The above $G$ is the desired $W$-graph for our graph structure on $M(\Gamma) . \Gamma$ can be recovered from $G$.

Proof. That $\Gamma$ can be recovered from $G$ is clear, since a rational number $p / q>1$ has a unique continued fraction expansion $p / q=\left[b_{1}, \ldots, b_{k}\right]$ with $b_{i} \geqslant 2$.

To see that $G$ is correctly described, observe first that the pieces being pasted together in plumbing come with trivializations of the bundle structures on their boundaries. For any edge $\sigma$ of $G$ this determines candidates for the bases $\left\{\mu_{\sigma}^{ \pm}, \nu_{\sigma}^{ \pm}\right\}$ which we need to compute $\left(\alpha_{\sigma}, \beta_{\sigma}\right)$. In most cases these candidates are incorrect and we must adjust $\nu_{\sigma}^{ \pm}$by a multiple of $\mu_{\sigma}^{ \pm}$to correct them. This corresponds to a change of boundary trivializations which will lead to the adjustment of the cross-section obstructions described in step 3. We shall denote the uncorrected bases coming from our given trivializations by $\left\{\mu_{\sigma}^{ \pm}, \lambda_{\sigma}^{ \pm}\right\}$.

The computations for the two types of chain in step 1 are essentially identical, so we just consider a chain of the second type. By Lemmas 5.2 and 5.3 this chain leads to the pasting $M=\left(\ldots \cup E_{n}\right) \cup_{C}\left(E_{m} \cup \ldots\right)$ where $C: S^{1} \times S^{1} \rightarrow S^{1} \times S^{1}$ has matrix

$$
C=(-1)^{c}\left(\begin{array}{cc}
q & p \\
-q^{\prime} & -p^{\prime}
\end{array}\right), \quad p / q=\left[b_{1}, \ldots, b_{k}\right], \quad q p^{\prime}-p q^{\prime}=1 .
$$

and $c$ is the number of (-)-edges of the chain. Our notation is not meant to suggest that $n$ and $m$ must be distinct vertices. Thus, putting $\varepsilon=(-1)^{c}$, we get $C\left(t^{\varepsilon p}, t^{-\varepsilon q}\right)=(1, t)$, or in other words $\mu_{\sigma}^{+}=\varepsilon\left(p \lambda_{\sigma}^{-}-q \mu_{\sigma}^{-}\right)$. Now $\nu_{\sigma}^{-}=\lambda_{\sigma}^{-}-l \mu_{\sigma}^{-}$ for some $l \in \mathbf{Z}$, and this gives $\mu_{\sigma}^{+}=\varepsilon\left(p \nu_{\sigma}^{-}+(l p-q) \mu_{\sigma}^{-}\right)=\varepsilon\left(\alpha_{\sigma} \nu_{\sigma}^{-}+\beta_{\sigma} \mu_{\sigma}^{-}\right)$. To satisfy $0<\beta_{\sigma}<\alpha_{\sigma}$ we must thus have $l=1$, giving

$$
\nu_{\sigma}^{-}=\lambda_{\sigma}^{-}-\mu_{\sigma}^{-}, \quad\left(\alpha_{\sigma}, \beta_{\sigma}\right)=(p, p-q), \quad \varepsilon_{\sigma}=\varepsilon .
$$


The same computation with the chain reversed shows $\nu_{\sigma}^{+}=\lambda_{\sigma}^{+}-\mu_{\sigma}^{+}$. These changes of trivialization in $\partial E_{n}$ and $\partial E_{m}$ have the effect of adding 1 to the cross-section obstructions $e_{n}$ and $e_{m}$ (or adding 2 to $e_{n}$ if $n=m$ ). That the adjustment is by +1 rather than -1 can be computed directly, but is seen more easily by running through the same argument with a reducible chain

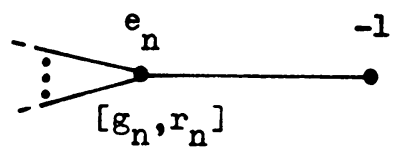

Thus step 1 is proved. Step 2 is trivial and the corresponding $\varepsilon_{\sigma}$ is just the corresponding edge sign. Moreover step 2 leads to no cross-section adjustment, so step 3 is proved. Collecting the above information on the signs $\varepsilon_{\sigma}$ proves step 4.

The following graph $G$ will be important below:

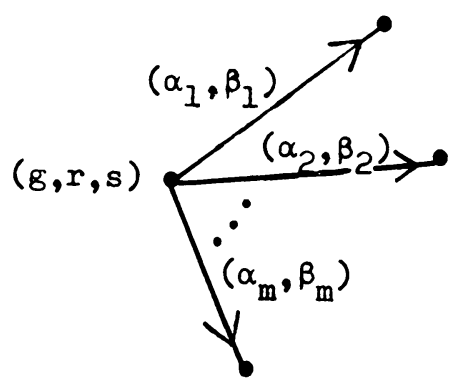

If $g \geqslant 0$ and $r=0$ then $G$ describes the natural graph structure on the closed Seifert manifold with orientable base with Seifert invariants $(g ;-s$; $\left.\left(\alpha_{1}, \beta_{1}\right), \ldots,\left(\alpha_{m}, \beta_{m}\right)\right)$. For general $g$ and $r$ it still describes the natural graph structure on an oriented Seifert manifold, with possibly nonorientable base, whose Seifert invariants can be written, with a minor alteration of standard notation, as $\left((g, r) ;-s ;\left(\alpha_{1}, \beta_{1}\right), \ldots,\left(\alpha_{m}, \beta_{m}\right)\right)$. We thus call any graph $G$ as above a Seifert manifold graph, and the corresponding graph structure is called a Seifert graph structure. We call the corresponding plumbing graph star-shaped.

We are now ready to prove Theorem 4.2. Let $\Gamma$ be a plumbing graph in normal form, which by 4.3 we can assume to be connected. If $\Gamma$ is a straight line graph or a cyclic graph we shall show in the next section that $M(\Gamma)$ is then respectively a lens space $L(p, q)$ (we include the cases $L(1,0)=S^{3}$ and $L(0,1)=S^{1} \times S^{2}$ ) or a torus bundle $T(A)$ with $\mid$ trace $A \mid \geqslant 3$ and that the straight line or cyclic graph $\Gamma$ is determined by $M(\Gamma)$. We thus exclude these special cases for now and must thus show that, with this exclusion:

(i) $M(\Gamma)$ is neither a lens space nor a torus bundle $T(A)$ with $\mid$ trace $A \mid \geqslant 3$;

(ii) the $W$-graph $G$ constructed above is determined by (the oriented homeomorphism type of) $M(\Gamma)$.

Theorem 4.2 then follows from Theorem 5.6.

We first show (ii). Call two graph structures on $M$ equivalent if they are related by an orientation preserving homeomorphism of $M$. Waldhausen [24, Theorem 8.1] 
shows that if $M$ has two inequivalent reduced graph structures then one of the following four cases applies:

1. Each graph structure is described by a Seifert manifold graph with $g=0$, $r=0, m \leqslant 3$ or $g=-1, r=0, m \leqslant 1$.

2. $M$ is a torus bundle $T(A)$ and one graph structure is the canonical graph structure (that is a fiber, so $|\operatorname{trace} A| \neq 2$ by reducedness) and the other a Seifert graph structure with $g=0, r=0, m \leqslant 3$.

3. The two graph structures are given by the $W$-graphs

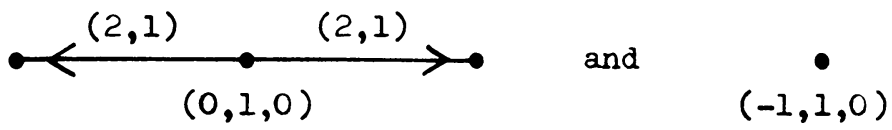

4. The two graph structures are given by the $W$-graphs

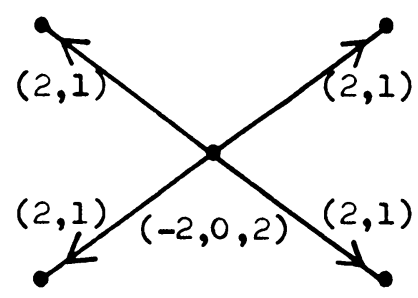

and

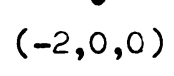

Now by condition $\mathrm{N} 5$ for normal form, the reduced graph structure we have constructed is never a Seifert graph structure with $g=-1, r=0$ and $m \leqslant 1$. Also by Orlik, Vogt and Zieschang [20] the Seifert invariants of a closed Seifert manifold $M$ with orientable base are uniquely determined by $M$, unless $M$ is a lens space and $m \leqslant 2$. Thus nonuniqueness of type 1 cannot occur for our reduced graph structure. Case 2 cannot occur by our exclusion of cyclic graphs. Cases 3 and 4 cannot occur, since by condition N6 for normal form we always take the left-hand graph structure in these cases.

Thus (ii) is proved. To prove (i), suppose first that $M(\Gamma)$ is a torus bundle $T(A)$ with $\mid$ trace $A \mid \geqslant 3$. Since such a torus bundle also has its canonical graph structure, this could only happen if nonuniqueness of type 2 above applied. But the only torus bundles which admit a Seifert manifold structure are those with $|\operatorname{trace} A| \leqslant 2$ (see for instance Orlik [18]), so this cannot happen. Thus suppose $M(\Gamma)$ were a lens space. Then $M(\Gamma) \neq S^{1} \times S^{2}$ since Waldhausen shows that reduced graph manifolds are irreducible. Thus $M(\Gamma)=L(p, q)$ with $p \neq 0$, so in particular $M(\Gamma)$ is not sufficiently large. But Waldhausen's Lemma 7.3 then implies that the $W$-graph $G$ corresponding to $\Gamma$ must be a Seifert manifold graph. On the other hand, by the classification of Seifert manifolds ([19], [20], see also [16] where a minor gap is corrected), the only Seifert manifold structures on lens spaces are those with $g=0$, $r=0, m \leqslant 2$, which do not yield reduced graph structures, or those given by the $W$-graphs

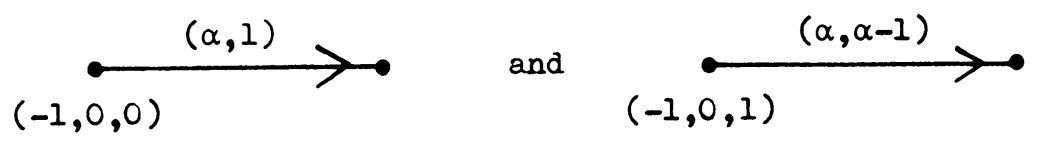


which yield respectively $L(4 \alpha, 2 \alpha-1)$ and $L(4 \alpha, 2 \alpha+1)$. Since graphs of the latter type are excluded by condition N5 of normal form, the proof is complete.

The following corollary will be useful later.

COROLlaRY 5.7. Suppose $\Gamma$ is a connected normal form plumbing graph and $M(\Gamma)$ is a Seifert manifold which is not a lens space. Then $\Gamma$ is the natural star-shaped plumbing graph

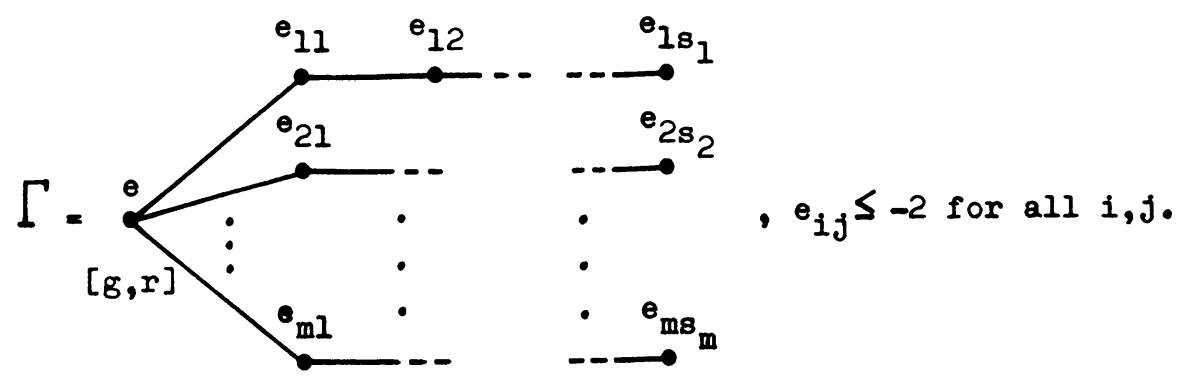

of a Seifert manifold structure on $M$. Moreover, if $M(\Gamma)$ admits a Seifert structure with orientable base, then $\Gamma$ corresponds to this Seifert structure. The Seifert invariants are $\left((g, r) ;-e-m ;\left(\alpha_{1}, \beta_{1}\right), \ldots,\left(\alpha_{m}, \beta_{m}\right)\right)$ with $\alpha_{i} /\left(\alpha_{i}-\beta_{i}\right)=$ $\left[-e_{i 1}, \ldots,-e_{i s_{i}}\right]$.

Proof. If $M(\Gamma)$ is a Seifert manifold with Seifert invariants as described above, then it has a plumbing graph as above. Either this plumbing graph is in normal form, in which case it is $\Gamma$ by uniqueness; this is always so if $g \geqslant 0$. Otherwise it is not in normal form, in which case, step 5 or one of the last two cases of step 6 of reduction to normal form replaces $\Gamma$ by the graph for a different Seifert structure on $M(\Gamma)$ which has orientable base.

6. Lens space and torus bundles. The part of 4.2 remaining to be proved is included in the following theorem.

THEOREM 6.1. The following normal form plumbing graphs give all lens spaces and all torus bundles. In each case the graph $\Gamma$ is the unique normal form plumbing graph for the corresponding manifold.

I. $\Gamma=\varnothing ; M(\Gamma)=S^{3}=L(1,0)$.

II.

$$
\Gamma=\stackrel{0}{ }
$$

$M(\Gamma)=S^{1} \times S^{2}=L(0,1)$.

III.

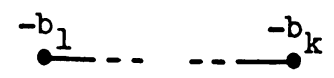

$k \geqslant 1, b_{i} \geqslant 2$ for all $i ; M(\Gamma)=L(p, q)$ with $p / q=\left[b_{1}, \ldots, b_{k}\right]$.

IV.

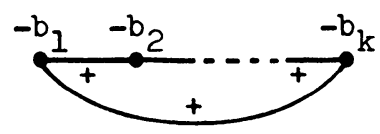


$b_{i} \geqslant 2$ for all $i, b_{i} \geqslant 3$ for some $i ; M(\Gamma)=T(A), A=A\left(b_{1}, \ldots, b_{k}\right)$, trace $A \geqslant 3$. $\mathrm{V}$.

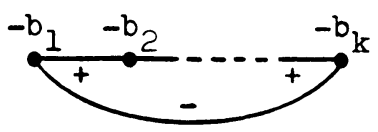

$b_{i} \geqslant 2$ for all $i, b_{i} \geqslant 3$ for some $i ; M(\Gamma)=T(A), A=-A\left(b_{1}, \ldots, b_{k}\right)$, trace $A$ $\leqslant-3$.

VI. $\Gamma$ one of the Seifert manifold graphs on the right in $\mathrm{R} 7$ of $\S 2 ; M(\Gamma)=T(A)$ with $A$ as described in $\S 2,|\operatorname{trace} A|<2$.

VII.

$$
\Gamma=\frac{-b}{[1]}
$$

$M(\Gamma)=T(A), A=\left(\begin{array}{ll}1 & 9 \\ b & 1\end{array}\right)$.

VIII.

$$
\Gamma=\stackrel{-b}{\cdot-2]}
$$

$b \neq 0 ; M(\Gamma)=T(A), A=-\left(\begin{array}{ll}1 & 9 \\ b & 1\end{array}\right)$.

IX.

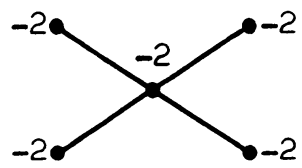

$M(\Gamma)=T(A), A=-\left(\begin{array}{ll}1 & 9 \\ 0 & 1\end{array}\right)$. If

PROOF. We first check that $M(\Gamma)$ is correctly described for each of the above $\Gamma$.

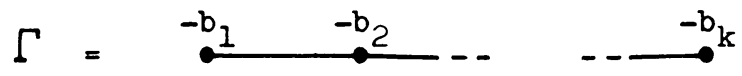

with no condition on the $b_{i}$, then the computation preceding Lemma 5.3 shows $M(\Gamma)=S^{1} \times D^{2} \cup_{B} S^{1} \times D^{2}$ with $B=A\left(b_{1}, \ldots, b_{k}\right) J=\left(\begin{array}{cc}q & p \\ -q^{\prime} & -p^{\prime}\end{array}\right)$ with $p / q=$ $\left[b_{1}, \ldots, b_{k}\right]$ and $p^{\prime} q-p q^{\prime}=1$, which is a standard description of $L(p, q)$. Thus $\mathrm{I}$, II, and III are verified.

If

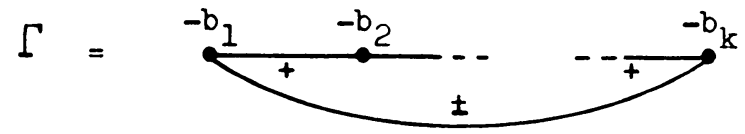

with no condition on the $b_{i}$, then a similar computation shows $M(\Gamma)=T(A)$ with $A= \pm A\left(b_{1}, \ldots, b_{k}\right)$. Thus, cases IV and V are clear, while cases VI-IX also follow, since those graphs are equivalent by a handle extrusion to suitable (nonnormal form) cyclic graphs. The statements about $\operatorname{trace}(A)$ in cases IV and $\mathrm{V}$ follow from Lemma 5.1 and a trivial induction. 
We have already seen in $\$ 5$ that a normal form plumbing graph for a lens space $L(p, q)$ must be a straight line graph $\Gamma$. The uniqueness statement for $\Gamma$ is proved in [7]. In fact if $0 \leqslant q<p$ and $0 \leqslant q^{\prime}<p$, then $L(p, q) \cong L\left(p, q^{\prime}\right)$ if and only if $q=q^{\prime}$ or $q q^{\prime} \equiv 1(\bmod p)$, and in [7] it is shown that if $p / q=\left[b_{1}, \ldots, b_{k}\right]$ is the unique continued fraction with $b_{i} \geqslant 2$, then $p / q^{\prime}=\left[b_{k}, \ldots, b_{1}\right]$ is the corresponding one for $p / q^{\prime}$ with $q q^{\prime} \equiv 1(\bmod p)$.

To see the corresponding statements for the torus bundle cases, we need the following well-known and easy lemma.

LEMMA 6.2. $T(A) \cong T(B)$ if and only if $A$ is conjugate in $\operatorname{SL}(2, \mathbf{Z})$ to $B$ or $J B^{-1} J^{-1}$, where $J=\left(\begin{array}{ll}0 & 1 \\ 1 & 0\end{array}\right)$.

Now the matrices $A$ occurring in cases IV-IX of 6.1 include all conjugacy classes of matrices with $|\operatorname{trace} A| \leqslant 2$, and the uniqueness of $\Gamma$ in these cases was proved in $\S 5$. Thus only cases IV and $\mathrm{V}$ need proof. Since the classification of conjugacy classes of $A \in \operatorname{SL}(2, \mathbf{Z})$ with $\operatorname{trace}(A)<0$ is equivalent to the classification for $\operatorname{trace}(A)>0$ (by multiplying by -1 ), cases IV and $\mathrm{V}$ are equivalent to each other, so we need only consider case IV. A trivial computation shows

$$
A\left(b_{k}, \ldots, b_{1}\right)=J A\left(b_{1}, \ldots, b_{k}\right)^{-1} J^{-1},
$$

so the following proposition completes the proof of 6.1. Define an oriented cycle to be a tuple $\left(\left(b_{1}, \ldots, b_{k}\right)\right)$ of integers $b_{i} \geqslant 2$ with some $b_{i} \geqslant 3$, where we consider two cycles to be the same if they are related by a cyclic permutation of the indices. For $A \in \mathrm{SL}(2, \mathbf{Z})$ let $(A)$ be its conjugacy class.

Proposition 6.3. The mapping $\left(\left(b_{1}, \ldots, b_{k}\right)\right) \mapsto\left(A\left(b_{1}, \ldots, b_{k}\right)\right)$ is a bijection from the set of oriented cycles to the set of conjugacy classes of $A \in \operatorname{SL}(2, Z)$ with trace $A \geqslant 3$.

Proof. See [6] or [9]. For completeness we describe how to find the cycle $\left(\left(b_{1}, \ldots, b_{k}\right)\right)$, given $A$. If one has never seen it, it is a pleasant exercise to fill in the proof oneself. Given $A \in \operatorname{SL}(2, \mathrm{Z})$ with $t=\operatorname{trace}(A)>3$ we consider the Möbius transformation on $\mathbf{R} \cup(\infty)$ given by

$$
x \mapsto \frac{a x+c}{b x+d}, \quad A=\left(\begin{array}{ll}
a & b \\
c & d
\end{array}\right)
$$

(note the transposition). Let $\omega \in \mathbf{R}$ be the stable fixed point of this map. Direct computation shows

$$
\omega=\left(\left(a-d+\sqrt{t^{2}-4}\right) / 2 b\right.
$$

Since $\omega$ is a quadratic irrationality, its infinite continued fraction expansion (with minus signs, as in $\S 5) \omega=\left[a_{0}, a_{1}, \ldots\right], a_{i} \geqslant 2$ for $i \geqslant 1$, is ultimately periodic. Let $\left(c_{1}, \ldots, c_{s}\right)$ be a primitive (that is shortest) period, and put $B=A\left(c_{1}, \ldots, c_{s}\right)$. If $t_{0}=\operatorname{trace}(B)$, then the desired cycle is

$$
\left(b_{1}, \ldots, b_{k}\right)=\left(c_{1}, \ldots, c_{s}, c_{1}, \ldots, c_{s}, \ldots, \ldots, c_{s}\right)
$$

( $n$ repeats), where $n$ is found from the relation $\operatorname{trace}\left(B^{n}\right)=\operatorname{trace} A$, or equivalently

$$
\left(t+\sqrt{t^{2}-4}\right) / 2=\left[\left(t_{0}+\sqrt{t_{0}^{2}-4}\right) / 2\right]^{n} \text {. }
$$


7. Orientation reversal. Let $\Gamma$ be a connected normal form plumbing graph. We wish to describe the normal form plumbing graph for $-M(\Gamma)$. We must recall some notation.

If $i$ is a vertex of $\Gamma$ which is not on a chain of $\Gamma$, then $i$ corresponds to a vertex of the corresponding $W$-graph $G$ and we define an integer $c_{i}$ as in step 3 preceding Theorem 5.6, that is, $c_{i}$ is the degree of vertex $i$ in the subgraph $G_{0}$ of $G$ obtained by deleting $(1,0)$-weighted edges. Equivalently, $c_{i}$ is the number of maximal chains of positive length in $\Gamma$ which have vertex $i$ as an endpoint, with chains which both begin and end at $i$ counted twice.

THEOREM 7.1. If $\Gamma$ is a connected normal form plumbing graph which is not a cyclic or straight line graph, then the normal form plumbing graph $\Gamma^{\prime}$ for $-M(\Gamma)$ is obtained from $\Gamma$ as follows:

(1) If $i$ is a vertex which is not on a chain and $r_{i}=0$, replace $e_{i}$ by $-e_{i}-c_{i}$.

(2) Replace any maximal chain
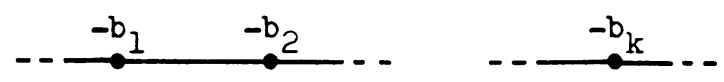

or
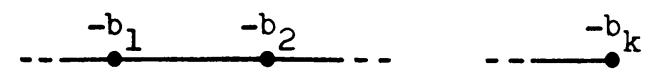

of $\Gamma$ by the chain
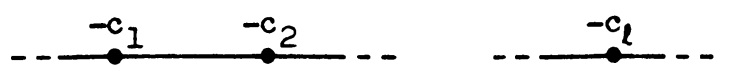

or
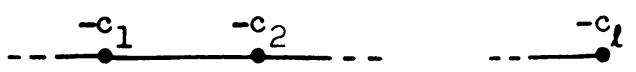

where the $c_{i}$ are determined as follows. If

$$
\left(b_{1}, \ldots, b_{k}\right)=(\underbrace{2, \ldots, 2}_{n_{0}}, m_{1}+3, \frac{2, \ldots, 2}{n_{1}}, m_{2}+3, \ldots, m_{s}+3, \underbrace{2, \ldots, 2}_{n_{s}})
$$

with $n_{i} \geqslant 0$ and $m_{i} \geqslant 0$, then

$$
\begin{aligned}
& \left(c_{1}, \ldots, c_{l}\right) \\
& \quad=(n_{0}+2, \underbrace{2, \ldots, 2}_{m_{1}}, n_{1}+3, \underbrace{2, \ldots, 2}_{m_{2}}, \ldots, n_{s-1}+3, \underbrace{2, \ldots, 2}_{m_{s}}, n_{s}+2) .
\end{aligned}
$$

(3) Via the obvious identification $H_{1}\left(\Gamma^{*}\right)=H_{1}\left(\left(\Gamma^{\prime}\right)^{*}\right)$, the homomorphism $\varepsilon_{\Gamma^{\prime}}$ : $H_{1}\left(\left(\Gamma^{\prime}\right)^{*}\right) \rightarrow \mathrm{Z} / 2$ is given as $\varepsilon_{\Gamma^{\prime}}=\varepsilon_{\Gamma}+\varepsilon_{0}$, where $\varepsilon_{0}$ assigns to any cycle of $\left(\Gamma^{\prime}\right)^{*}$ the number modulo 2 of maximal chains on this cycle.

Moreover, if $G$ and $G^{\prime}$ are the $W$-graphs corresponding to $\Gamma$ and $\Gamma^{\prime}$, so $G^{\prime}$ describes - $M(\Gamma)$, then $G^{\prime}$ is obtained from $G$ as follows:

$\left(1^{\prime}\right)$ For any vertex $i$ with $r_{i}=0$ replace $s_{i}$ by $-s_{i}+c_{i}$.

$\left(2^{\prime}\right)$ Edge weights $(1,0)$ are unchanged, otherwise replace $(\alpha, \beta)$ by $(\alpha, \alpha-\beta)$.

$\left(3^{\prime}\right) \varepsilon: H_{1}\left(G^{*}\right) \rightarrow \mathbf{Z} / 2$ is replaced by $\varepsilon+\varepsilon_{0}$, where $\varepsilon_{0}$ assigns to any cycle of $G^{*}$ its length modulo 2. 
Proof. Suppose we have a chain
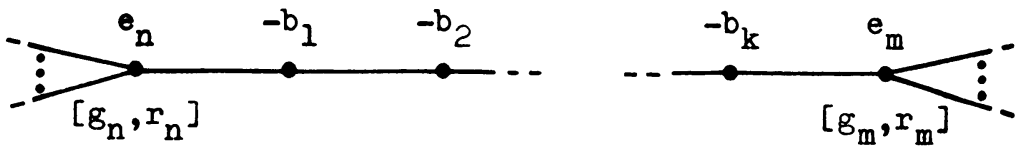

in $\Gamma$, where for the moment we assume $n \neq m, k \geqslant 1$, and we disregard edge signs. In the graph $-\Gamma$ (see Proposition 3.3) this becomes
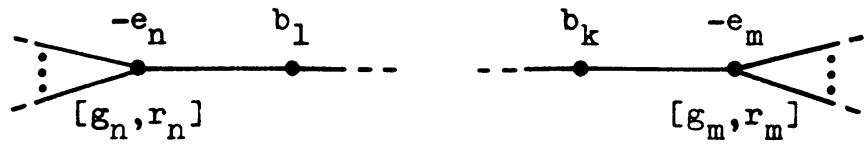

Using the notation of (2) above, we abbreviate the relevant information as

$$
(-e_{n}, \frac{2, \ldots, 2}{n_{0}}, m_{1}+3, \ldots, m_{s}+3, \underbrace{2, \ldots, 2}_{n_{s}},-e_{m}) \text {, }
$$

which we abbreviate further as $\left(-e_{n}, n_{0} \cdot 2, m_{1}+3, \ldots, m_{s}+3, n_{s} \cdot 2,-e_{m}\right)$. Now alter this by blowing up and blowing down as follows. After $(s+1)$ $(-1)$-blow-ups we have (with a suitable reinterpretation if some $n_{i}=0$ )

$$
\begin{aligned}
\left(-e_{n},\left(n_{0}-1\right) \cdot 2,1,-1, m_{1}+2, \ldots,\left(n_{s-1}-1\right) \cdot 2,\right. \\
\left.1,-1, m_{s}+2,\left(n_{s}-1\right) \cdot 2,1,-1,-e_{m}-1\right) .
\end{aligned}
$$

Now do $n_{0}+n_{1}+\cdots+n_{s}(+1)$-blow-downs to get

$$
\begin{aligned}
\left(-e_{n}-1,-n_{0}-1, m_{1}\right. & +1,-n_{1}-1, \ldots, \\
& \left.-n_{s-1}-1, m_{s}+1,-n_{s}-1,-e_{m}-1\right) .
\end{aligned}
$$

Next, doing $m_{i}(-1)$-blow-ups directly to the left of $\left(m_{i}+1\right)$ for each $i$ gives (again with suitable interpretation if some $m_{i}=0$ )

$$
\begin{aligned}
\left(-e_{n}-1,-n_{0}-2,\left(m_{1}-1\right) \cdot(-2),-1,1,-n_{1}-2, \ldots,\right. \\
\left.\left(m_{s}-1\right) \cdot(-2),-1,1,-n_{s}-1,-e_{m}-1\right),
\end{aligned}
$$

after which blowing down the $s(+1)$ 's gives

$$
\begin{gathered}
\left(-e_{n}-1,-n_{0}-2, m_{1} \cdot(-2),-n_{1}-3, \ldots,-n_{s-1}-3,\right. \\
\left.m_{s}(-2),-n_{s}-2,-e_{m}-1\right) \\
=\left(-e_{n}-1,-c_{1},-c_{2}, \ldots,-c_{l},-e_{m}-1\right) .
\end{gathered}
$$

If $k=0$, then much more trivially, the final result is $\left(-e_{n}, e_{m}\right)$ in the above shorthand. A similar analysis applies if $n=m$ and for maximal chains of the second type, so parts (1) and (2) of the theorem follow.

Now let us consider the effect on edge signs of the above reduction. Our initial chain had $n_{0}+1+n_{1}+1+\cdots+n_{s}=\sum n_{i}+s$ vertices, hence $\sum n_{i}+s+1$ edges, so the replacement of $\Gamma$ by $-\Gamma$ reverses $\left(\sum n_{i}+s+1\right)$ edge signs on this chain. Now a (-1)-blow-up or blow-down does not alter the number of (-)-edges modulo 2 on the chain while a $(+1)$-blow-down alters this number by 1 . Since our process involved $\sum n_{i}(+1)$-blow-ups and $s(+1)$-blow-downs, the total number of 
(-)-edges of the chain has been altered by $\left(\sum n_{i}+s+1\right)+\sum n_{i}+s \equiv 1(\bmod 2)$. Hence (3) follows.

The statements about $G^{\prime}$ can be verified directly from the description of a $W$-graph in terms of the graph structure, but follow more easily by applying Theorem 5.6 to $\Gamma^{\prime}$. Namely, since $s_{i}=e_{i}+c_{i}, s_{i}$ gets replaced in $G^{\prime}$ by $\left(-e_{i}-c_{i}\right)$ $+c_{i}=-e_{i}=-s_{i}+c_{i}$, proving $\left(1^{\prime}\right)$. Statement $\left(3^{\prime}\right)$ is immediate from (3) and Theorem 5.6. Statement $\left(2^{\prime}\right)$ follows from 5.6 and the following lemma.

LEMMA 7.2. If $p / q=\left[b_{1}, \ldots, b_{k}\right]$ with $b_{i} \geqslant 2$, then $p /(p-q)=\left[c_{1}, \ldots, c_{l}\right]$, with $c_{i}$ as in Theorem 7.1 (2).

This can be proved directly, but we sketch an alternative method. The following relations are easily verified for continued fractions:

$$
\begin{aligned}
{\left[-x_{1},-x_{2}, \ldots,-x_{k}\right] } & =-\left[x_{1}, \ldots, x_{k}\right], \\
{\left[x_{1}+n, x_{2}, \ldots, x_{k}\right] } & =\left[x_{1}, \ldots, x_{k}\right]+n, \\
{\left[x_{1}, \ldots, x_{i}, x_{i+1}, \ldots, x_{k}\right] } & =\left[x_{1}, \ldots, x_{i} \pm 1, \pm 1, x_{i+1} \pm 1, \ldots, x_{k}\right], \\
{\left[ \pm 1, x_{1}, \ldots, x_{k}\right] } & = \pm 1-1 /\left[x_{1}, \ldots, x_{k}\right] .
\end{aligned}
$$

The only nontrivial one is the third, which is a trivial verification when $i=1$ and $k=2$, and follows in general from this case, since $\left[x_{1}, \ldots, x_{i},\left[x_{i+1}, \ldots, x_{k}\right]\right]=$ $\left[x_{1}, \ldots, x_{i}, x_{i+1}, \ldots, x_{k}\right]$. Lemma 7.2 now follows rapidly by analyzing the effect of the moves used in the proof of part (1) of 7.1 above to reduce $\left(b_{1}, \ldots, b_{k}\right)$ to $\left(-c_{1}, \ldots,-c_{l}\right)$.

THEOREM 7.3. For straight line and cyclic graphs $\Gamma$ in normal form, the normal form graph $\Gamma^{\prime}$ for $-M(\Gamma)$ is as follows. If

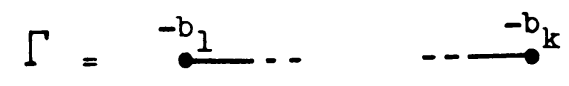

then

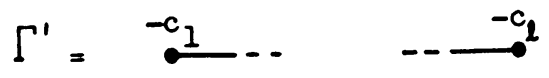

with $c_{1}, \ldots, c_{l}$ as in Theorem 7.1. If

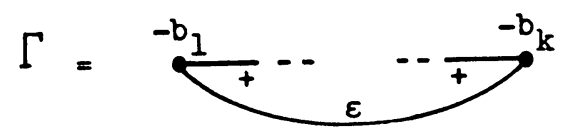

then

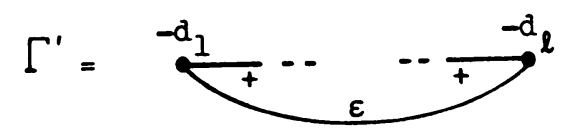

where, if

$$
\left(\left(b_{1}, \ldots, b_{k}\right)\right)=\left((m_{1}+3, \underbrace{2, \ldots, 2}_{n_{1}}, m_{2}+3, \ldots, m_{s}+3, \underbrace{2, \ldots, 2}_{n_{s}})\right),
$$


then

$$
\left(\left(d_{1}, \ldots, d_{k}\right)\right)=\left((n_{s}+3, \frac{2, \ldots, 2}{m_{s}}, n_{s-1}+3, \ldots, n_{1}+3, \underbrace{2, \ldots, 2}_{m_{1}})\right) .
$$

Proof. For $\Gamma$ a straight line graph this is Lemma 7.2. For cyclic $\Gamma$ the proof is essentially the same computation as for part (1) of 7.1.

8. Resolution of singularities and their normal form graph. Let $(V, p)$ be the germ of an isolated normal complex surface singularity. We denote its link (the boundary of a regular neighborhood of $p$ in $V$ ) by $M^{3}=M(V, p)$. Let $\pi: \tilde{V} \rightarrow V$ be the minimal good resolution of $(V, p)$. That is, $\pi$ is a proper holomorphic map such that:

(i) $\pi \mid \tilde{V}-\pi^{-1}(p) \rightarrow V-p$ is biholomorphic.

(ii) All singularities of the exceptional divisor $K=\pi^{-1}(p)$ are normal crossings.

(iii) (Minimality.) If a nonsingular irreducible component $K_{i}$ of $K$ has genus 0 and self-intersection number $K_{i} \cdot K_{i}=-1$, then it has at least three intersection points with the rest of $K$.

If (iii) were contravened, one could blow down the corresponding $K_{i}$, keeping the resolution good. Topologically, statement (ii) says that $K$ is the union $K=K_{1}$ $\cup \cdots \cup K_{k}$ of immersed closed connected real surfaces $K_{i}$ having transverse self-intersections and transverse mutual intersections, and no three-fold intersections.

The topology of the resolution can be coded in the dual graph $\Delta=\Delta(V, p)$ (see for instance [7]). The vertices of $\Delta$ are labelled $1, \ldots, k$ and are each weighted by the integers $e_{i}=e\left(\nu\left(K_{i}\right)\right.$ ) (the euler number of the normal bundle of $K_{i}$ in $\tilde{V}$ ) and $g_{i}=g\left(K_{i}\right)$ (the genus of $\left.K_{i}\right)$. $\Delta$ has an edge from $i$ to $j$ for each intersection point of $K_{i}$ and $K_{j}$ and an edge from $i$ to $i$ (that is a loop at $i$ ) for each self-intersection point of $K_{i}$.

We can consider $\Delta$ as a plumbing graph with all $r_{i}$ weights zero and all edge signs equal to + . The graph $\Delta$ determines the local topology of the resolution. Indeed if $P(\Delta)$ is the 4-manifold obtained by plumbing disc bundles according to $\Delta$ (as in [7] for example), so $\partial P(\Delta)=M(\Delta)$ is the 3-manifold obtained by plumbing in the sense of this paper, then $P(\Delta)$ is diffeomorphic to a regular neighborhood of $K=\pi^{-1}(p)$ in $\tilde{V}$ and $M(\Delta)$ is diffeomorphic to the link $M=M(V, p)$.

Denote by $S(\Delta)$ the intersection matrix $\left(K_{i} \cdot K_{j}\right)$. Thus $S(\Delta)=\left(a_{i j}\right)_{i, j=1, \ldots, k}$, with

$$
\begin{aligned}
a_{i j} & =e_{i}+2 \cdot(\text { number of loops at } i \text { in } \Delta), \quad i=j, \\
& =\text { number of edges from } i \text { to } j \text { in } \Delta, \quad i \neq j .
\end{aligned}
$$

It is well known that an oriented closed plumbing graph $\Delta$ (as defined in $\S 3$ ) is the dual graph of some (not necessarily minimal) good resolution of a singularity if and only if the edge signs are all + and $S(\Delta)$ is negative definite (Grauert [5]). Minimality of the resolution is equivalent to saying that $\Delta$ admits no $(-1)$-blowing down in the sense of plumbing graphs (see §4).

$S(\Delta)$ represents the intersection form on $H_{2}(P(\Delta))$ with respect to the basis represented by the $K_{i} \subset P(\Delta)$. If $\Delta_{0}$ is the full subgraph on some subset of the 
vertex set of $\Delta$, then $S\left(\Delta_{0}\right)$ represents the restriction of the intersection form to the corresponding subspace of $\mathrm{H}_{2}(P(\Delta))$, so it is also negative definite. In particular, all the $e_{i}$ weights of $\Delta$ are negative. Moreover, we have the following lemma.

LEMMA 8.1. If $\Delta$ is the dual graph of a minimal good resolution, then in any piece of $\Delta$ of the form

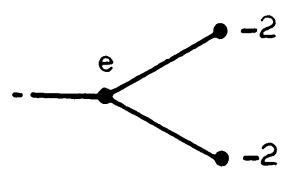

we have $e \leqslant-2$, and if

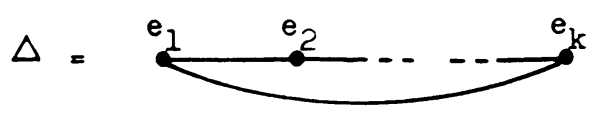

or

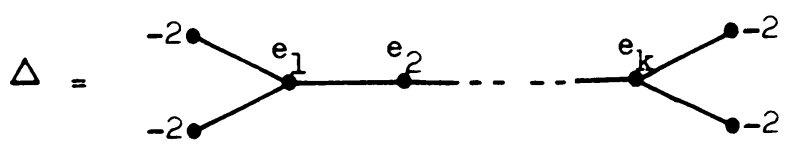

then $e_{i} \leqslant-2$ for all $i$, and some $e_{i} \leqslant-3$.

Proof. This is immediate from the above comments. Since
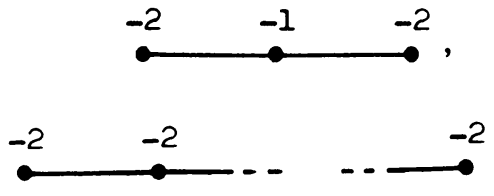

and

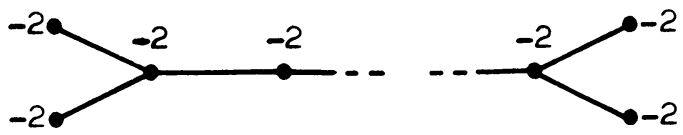

have indefinite forms.

THEOREM 8.2. The normal form $\Gamma$ of a resolution graph $\Delta$ is obtained as follows:

(1) If

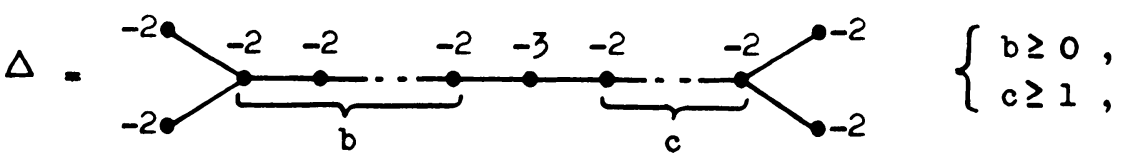

then

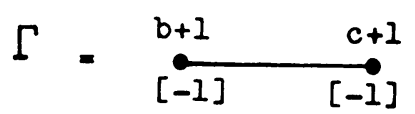


(2) If

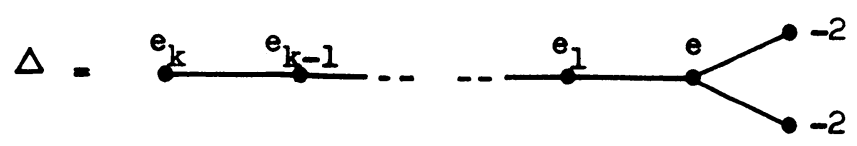

then $\Gamma=\Delta$.

(3) In all other cases $\Gamma$ is obtained from $\Delta$ by applying the following operations to $\Delta$ wherever possible:
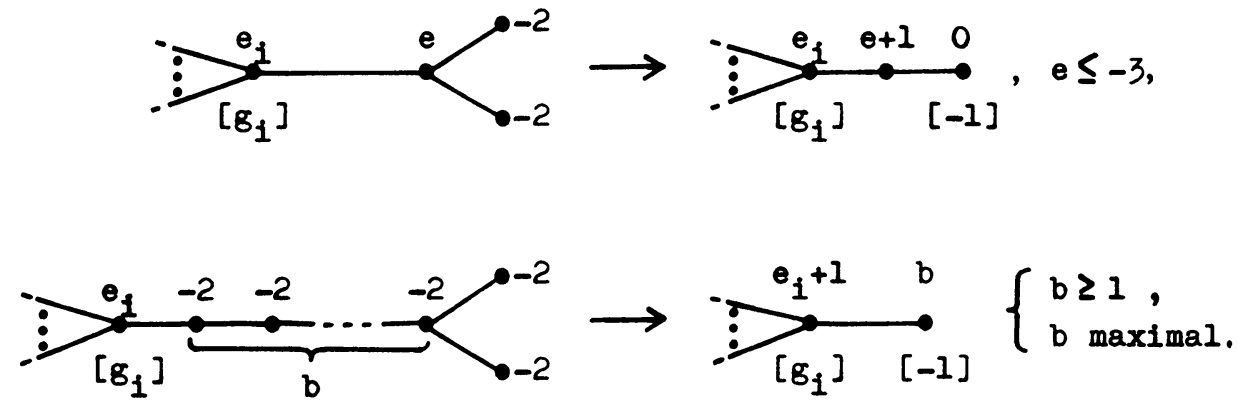

Moreover, $\Gamma$ has the following properties:

(i) All edge signs are +.

(ii) All genus weights satisfy $g_{i} \geqslant-1$.

(iii) Any vertex $i$ with $g_{i}=-1$ has degree 1 ; moreover it has euler number weight $e_{i} \geqslant 0$, and if $e_{i}=0$, then the maximal chain ending at $i$ has length $\geqslant 1$.

(iv) $\Delta$ is uniquely determined by $\Gamma$.

Proof. The proof that (1)-(3) reduce $\Delta$ to normal form is immediate from Lemma 8.1 and the definition of normal form. Note that (1) is given by two applications of (3); we have made a special case of (1) because it is the only case that an application of (3) can turn a chain of nonzero length ending in a vertex with $g_{i}=-1$ into a chain of zero length. With this remark, statement (iii) about $\Gamma$ becomes clear, while (i), (ii) and (iv) were already clear.

COROLlaRY 8.3. If the singularity link $M$ is a lens space, a torus bundle or a Seifert manifold, then the resolution graph $\Delta$ is already in normal form, so $\Delta=\Gamma$. All possible singularity links of these types are given by the following list:

(1) $M=L(p, q), p>1 ; \Gamma$ as in Theorem 6.1, III.

(2) $M=T(A)$, trace $A \geqslant 3$; $\Gamma$ as in Theorem 6.1, IV.

(3) $M=T(A), A$ conjugate to $\left(\begin{array}{ll}1 & 0 \\ b & 1\end{array}\right), b>0 ; \Gamma$ as in Theorem 6.1, VII.

(4) $M$ Seifert fibered with Seifert invariant $\left(g ; b ;\left(\alpha_{1}, \beta_{1}\right), \ldots,\left(\alpha_{m}, \beta_{m}\right)\right)$ with $g \geqslant 0$ and $m \geqslant 3$ if $g=0$, and $e(M)=-b-\sum \beta_{i} / \alpha_{i}<0 ; \Gamma$ as in Corollary 5.7.

PROof. For lens spaces and torus bundles this corollary is immediate by checking through the cases of Theorem 6.1, using negative definiteness of the intersection form $S(\Gamma)$. For Seifert manifolds the fact that $\Delta=\Gamma$ is by Corollary 5.7, while the statement about which Seifert manifolds occur is then the content of Theorem 5.2 of [16]. The number $e(M)$ is the euler number of a Seifert fibration, 
introduced in [16]. It is defined also for Seifert fibrations on lens spaces, but is an invariant of $M$ alone if $M$ is not a lens space, since then the Seifert fibration is unique up to homeomorphism.

9. Proofs of Theorems 1 to 7. By Theorems 8.2 and 5.1, any singularity link which is not a lens space has a reduced graph structure, and is thus irreducible by Waldhausen [24]. Since lens spaces $L(p, q)$ with $p \neq 0$ are also irreducible, Theorem 1 of the introduction follows.

Theorem 2 is immediate from Theorems 8.2 and 4.2, while Theorem 5 and Corollary 6 are included in Corollary 8.3 , plus the fact that any lens space $L(p, q)$ with $p>1$ admits a Seifert fibration with negative euler number (for instance $(0$; $(1,[p / q]),(q, p-q[p / q])))$.

For Theorem 3, note first that the "if" statement is trivial by Corollary 8.3 , since $-L(p, q)=L(p, p-q)$ and $-T(A)=T\left(A^{-1}\right)$. To see the converse suppose $M$ is a singularity link which is not a lens space or a torus bundle $T(A)$ with $\operatorname{trace}(A) \geqslant 3$ and suppose $-M$ is also a singularity link. Then $M$ cannot be a Seifert manifold, since otherwise $e(-M)=-e(M)$ would be positive, contradicting Corollary 8.3 (that $e(-M)=-e(M)$ is proved in [16], but also follows easily from Theorem 7.1). We shall reduce the general case to this case to obtain the desired contradiction.

Let $\Delta$ be the resolution graph for $M$ and $\Gamma$ its normal form, and let $\Delta^{\prime}$ and $\Gamma^{\prime}$ be the same for $-M$. By part 1 of Theorem 7.1 and statement (iii) in Theorem 8.2 we see $\Gamma$ can have no vertex $i$ with $g_{i}=-1$. By symmetry the same holds for $\Gamma^{\prime}$. Thus 8.2 implies that $\Gamma=\Delta$ and $\Gamma^{\prime}=\Delta^{\prime}$. Moreover, the fact that $\varepsilon_{\Gamma}$ and $\varepsilon_{\Gamma^{\prime}}$ are trivial implies, by part (3) of 7.1, that each cycle of $\Gamma$ is composed of an even number of maximal chains (and the same for $\Gamma^{\prime}$ ). In particular $\Gamma$ has no maximal chain which begins and ends at the same vertex. Now let $i$ be any vertex of $\Gamma$ which is not on a maximal chain. This exists, since by our exclusions, $\Gamma$ is not a straight line or cyclic plumbing graph. Consider the set of vertices of $\Gamma$ consisting of vertex $i$ together with all vertices on maximal chains which begin at $i$. Let $\Gamma_{0}$ be the full subgraph of $\Gamma$ on this set, so $\Gamma_{0}$ is a star-shaped plumbing graph and $M\left(\Gamma_{0}\right)$ is a Seifert manifold. Let $\Gamma_{0}^{\prime}$ be constructed similarly in $\Gamma^{\prime}$ starting from the vertex corresponding to $i$ in $\Gamma^{\prime}$. By Theorem 7.1 one sees that $\Gamma_{0}^{\prime}$ is precisely the normal form graph for $-M\left(\Gamma_{0}\right)$. But by the comments preceding Lemma 8.1 the intersection forms $S\left(\Gamma_{0}\right)$ and $S\left(\Gamma_{0}^{\prime}\right)$ are both negative definite. Thus both $M\left(\Gamma_{0}\right)$ and $-M\left(\Gamma_{0}\right)$ are singularity links, contradicting the case already proven.

Corollary 4 now follows, since by Waldhausen [25] a sufficiently large 3-manifold is determined up to orientation by its fundamental group and by Orlik, Vogt and Zieschang [20] the same holds for closed Seifert manifolds with orientable base which are not lens spaces. As remarked in $\$ 5$ these two cases cover all possibilities by Waldhausen [24, Lemma 7.3].

By Corollary 8.3 the only part of Theorem 7 needing proof is that if $M$ is a singularity link which fibers over $S^{1}$ then $M$ is a torus bundle. This is immediate from the classification in [14] of fibrations of graph manifolds, but is in fact much easier. Let $\Delta$ be an orientable closed plumbing graph with $S(\Delta)$ nonsingular 
(certainly true for our resolution graph) and let $f: M(\Delta) \rightarrow S^{1}$ be a fibration, whose fiber $F$ we can assume with no loss of generality to be connected. Now in general $H^{1}(M(\Delta), \mathbf{Z}) \cong H^{1}(\Delta ; \mathbf{Z}) \oplus \operatorname{Ker} S(\Delta)$ (see [7] or [21]), so in our case

$$
H^{1}(M(\Delta) ; \mathbf{Z})=H^{1}(\Delta ; \mathbf{Z}) \text {. }
$$

Since $H^{1}(M(\Delta) ; Z)=\left[M(\Delta), S^{1}\right]$, this tells us that any map $M(\Delta) \rightarrow S^{1}$ is homotopic to one which is induced in the natural way from a map $\Delta \rightarrow S^{1}$. In particular $f$ is homotopic to a map $g: M(\Delta) \rightarrow S^{1}$ such that for some regular value $p \in S^{1}$ the inverse image $g^{-1}(p)$ is a disjoint union of tori. It follows that the genus of the fiber $F$ of $f$ is at most 1, so in Theorem 7, $M$ can only be a torus bundle or an $S^{2}$-bundle. Irreducibility of $M$ eliminates fiber $S^{2}$, so the theorem follows.

10. Degenerating curves. Let $\pi: W \rightarrow D$ be a minimal good degenerating family of curves, as defined in the introduction. The singular fiber $K=\pi^{-1}(0)$ can be written as the union $K=K_{1} \cup \cdots \cup K_{k}$ of its irreducible components, and, exactly as in $\S 8$, the topology of the pair $(W, K)$ can be coded in the dual graph $\Delta$.

We can however put extra information into $\Delta$, namely the multiplicities $m_{i}$ of the components $K_{i}$. For the topologist these can be defined as follows. Note that $\mathrm{H}_{2}(W ; \mathrm{Z})$ is freely generated by the homology classes $\left[K_{i}\right]$ of the $K_{i}$. Thus if $F$ is a nonsingular fiber we have a homology relation

$$
[F]=m_{1}\left[K_{1}\right]+\cdots+m_{k}\left[K_{k}\right]
$$

which defines the $m_{i}$. It is easily seen that $m_{i}>0$ for all $i$. Note that the intersection numbers $[F] \cdot\left[K_{i}\right]$ are all zero. This is equivalent to the statement $S(\Delta)\left(m_{1}, \ldots, m_{k}\right)^{t}=0$, where $S(\Delta)$ is the intersection matrix, defined as in $\S 8$. It is easy to see that this equation can be solved for the $e_{i}$ weights, given the abstract graph $\Gamma$ and the $m_{i}$ weights, so the $m_{i}$ 's determine the $e_{i}$ 's. By Winters [26], the existence of $m_{i}>0$ such that the above equation holds is equivalent to $\Delta$ being the dual graph for a degenerating family of curves.

By a full subgraph $\Delta_{0}$ of $\Delta$ we mean a subgraph which includes every edge of $\Delta$ whose endpoints are in $\Delta_{0}$. Exactly the same proof as Mumford's proof that the intersection form for a resolution of a singularity is negative definite (see for instance [7]) proves the following well-known lemma.

LEMMA 10.1. If $\Delta_{0}$ is a connected full subgraph of $\Delta$ and $\Delta_{0} \neq \Delta$ then $S\left(\Delta_{0}\right)$ is negative definite. Hence $S(\Delta)$ is negative semidefinite, so $\left(m_{1}, \ldots, m_{k}\right)$ is determined up to a positive multiple by $S(\Delta)$.

Moreover it is well known that the euler number $\chi(F)$ of the nonsingular fiber is given by

$$
\chi(F)=\sum_{i=1}^{k} m_{i}\left(2-2 g_{i}-d_{i}\right)
$$

where $d_{i}$ is the degree of vertex $i$ (for a purely topological proof for arbitrary plumbed manifolds, see [14]). Thus $\Delta$ and $\chi(F)$ together determine the $m_{i}$. 
THEOREM 10.2. If $\Delta$ is the dual graph of a minimal good degenerating family then its normal form $\Gamma$ is obtained as follows.

(1) If

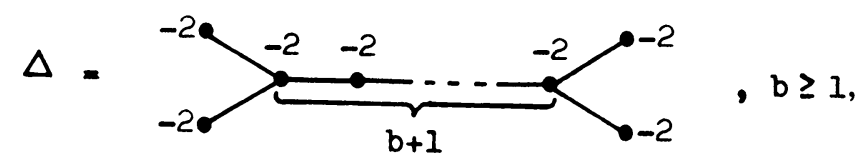

then

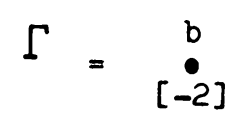

(2) If

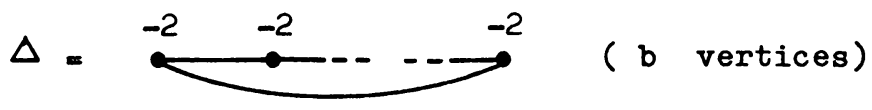

then

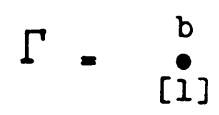

(3) Same as part (3) of Theorem 8.2.

Moreover, statements (i)-(iv) of Theorem 8.2 still hold except that in statement (ii) an exception must be made for the graph in (1) above.

Proof. Lemma 10.1 implies that the first statement of Lemma 8.1 still applies in the present situation. Moreover, by [16], the star-shaped graph $\Gamma$ of a Seifert manifold $M$ can have degenerate intersection form $S(\Gamma)$ if and only if $e(M)=0$, so graphs as in Theorem 8.2(2) cannot occur. Thus the proof of 10.2 is exactly like the proof of 8.2 .

COROLlaRY 10.3. The torus bundles which are links of degenerating families are those of cases VII and VIII of Theorem 6.1 with $b<0$ (in which case $\Delta$ is given by cases (1) and (2) of 10.2 above) and those of VI and IX in Theorem 6.1 (in which case $\Delta=\Gamma)$. The Seifert manifolds $M$ which are links of degenerating families are precisely those with $e(M)=0$, and $\Delta=\Gamma$ for these.

Proof. The statement about torus bundles (which is a result of Kodaira [12]) is proved exactly as in Corollary 8.3, by checking cases in 6.1. For Seifert manifolds the proof is implicit in the proof of Theorem 5.2 of [16], so we omit it.

The proofs of Theorem 8 and 9 and Corollary 10 of the introduction are now exactly like the proofs of the corresponding statements for singularity links. The only difference is that in proving Theorem 9 we assume $M$ is not a Seifert manifold and construct the subgraphs $\Gamma_{0}$ of $\Gamma$ and $\Gamma_{0}^{\prime}$ of $\Gamma^{\prime}$ as in the proof of Theorem 3, and it is Lemma 10.1 which gives the desired contradiction, since $\Gamma_{0}$ and $\Gamma_{0}^{\prime}$ are proper subgraphs of $\Gamma$ and $\Gamma^{\prime}$. 


\section{Appendix. Boundary framed graph manifolds and graph manifolds with meridians.}

1. Boundary framed graph manifolds. The boundary of a graph manifold (i.e. plumbed manifold) $M^{3}$ consists of tori. In many applications one has not just a graph manifold, but a graph manifold $M^{3}$ plus a specific diffeomorphism of each boundary component with $S^{1} \times S^{1}$. We call such a collection of diffeomorphisms a boundary framing on $M^{3}$. We wish to modify our classification to classify boundary framed graph manifolds.

A first attempt is as follows: as we described plumbing, the euler number weight $e_{i}$ at a vertex with $r_{i} \neq 0$ is irrelevant and should be omitted or disregarded; however if we retain this weight, then it determines specific framings of the corresponding boundary components of $M(\Gamma)$; thus one might try to work simply with plumbing graphs in which all vertices have euler number weights. However it is then hard to keep track of orientations at boundary components when applying moves of the plumbing calculus.

We therefore make the following definition: a decorated plumbing graph $\Gamma$ is a plumbing graph in the usual sense with the following modifications:

(i) Some vertices of degree 0 or 1 in $\Gamma$ are left unweighted. They are called boundary vertices, and are drawn in the graph as arrow heads (this is to conform with standard notation in resolution of singularities).

(ii) The $r_{i}$ weight at any weighted vertex is zero, hence omitted. Thus a typical such graph might look like:

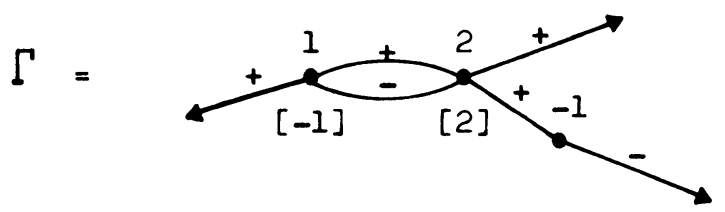

(where the genus at a vertex without genus weight is as usual understood to be zero).

We interpret the boundary vertices (arrowheads) as vertices with euler number and genus weights zero and $r$-weight equal to 1 . Thus the above graph $\Gamma$ should be considered a shorthand for

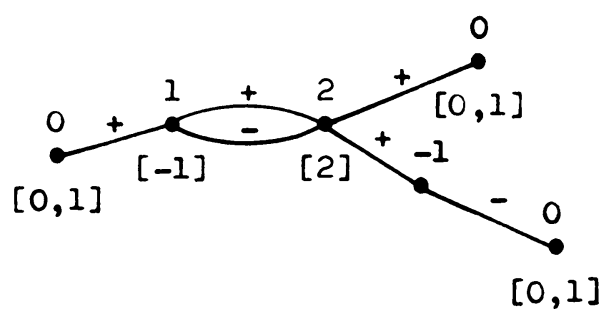

By our above remarks, with this interpretation, $\Gamma$ determines a well-defined boundary framed graph manifold. The graph $\boldsymbol{\Delta}$ consisting of a single arrowhead is also permitted, and represents a solid torus with obvious boundary framing.

The calculus for plumbing must be slightly modified for boundary framed plumbing via decorated plumbing graphs. 
R0 (altering edge weights) stays the same, except that it may of course not be applied to boundary vertices of $\Gamma$. Thus the information contained in the edge weights is completely coded by a homomorphism $\varepsilon_{\Gamma}: H_{1}\left(\Gamma^{* *}, B\right) \rightarrow \mathbf{Z} / 2$ defined as in $\S 2$, where $\Gamma^{* *}$ is the full subgraph of $\Gamma$ defined by all vertices $i$ with $g_{i} \geqslant 0$ and all boundary vertices, and $B$ is the set of boundary vertices. (Alternatively one could use the homomorphism $\varepsilon_{\Gamma}: H_{1}\left(\Gamma^{*}\right) \rightarrow \mathbf{Z} / 2$ of $\S 2$ plus a map $B \rightarrow\{+1,-1\}$, the latter well defined up to multiplication by -1 .)

R0-R7 stay the same except that moves which would alter the implied zero euler weight at boundary vertices are not permitted. That is, one cannot blow up or down or absorb a zero chain directly adjacent to a boundary vertex. The final reduction move $\mathrm{R} 8$ is deleted.

The definition of normal form stays the same except that vertices adjacent to boundary vertices are never considered as vertices on a chain in $\Gamma$ (and may thus have arbitrary euler weights), and a graph of the form

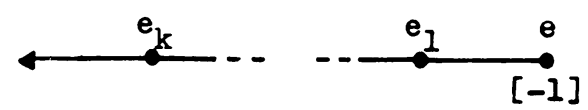

is not considered to be in normal form, while a graph of the form

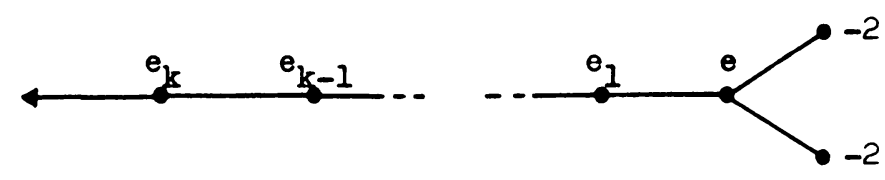

with $e_{i} \leqslant-2$ for $1 \leqslant i \leqslant k-1$ is considered to be in normal form (this modifies N3).

We call the resulting normal form F-normal form. The calculus for decorated plumbing graphs described above is called the $F$-calculus.

TheOREM. Any decorated plumbing graph can be reduced to a unique decorated plumbing graph in F-normal form by the F-calculus. Any boundary framed graph manifold has the form $M(\Gamma)$ for a unique decorated plumbing graph in $F$-normal form (up to isomorphism).

The proof is just like the proof for graph manifolds without boundary framings and therefore omitted.

2. Graph manifolds with meridians. In applications in algebraic geometry one usually has graph manifolds with something slightly less than a boundary framing, namely on each boundary component $T$ one just has a fibration $\pi: T \rightarrow S^{1}$ given. Equivalently (by taking fibers of $\pi$ ) an isotopy class of oriented simple closed curves on $T$ (called meridians) is given. The way this arises is as follows. Let $N^{3}$ be a closed graph manifold and let $K \subset N^{3}$ be a union of disjoint oriented embedded circles, such that the result $M^{3}$ of removing a small tubular neighborhood of $K$ is still a graph manifold. Then $M^{3}$ comes equipped with meridians in its boundary (namely meridians of the removed solid tori). Conversely any graph manifold $M^{3}$ with chosen meridians in its boundary (briefly: graph manifold with meridians) 
determines a unique way of pasting solid tori into the boundary components and hence determines a pair $(N, K)$ as above.

A decorated plumbing graph $\Gamma$ determines a graph manifold with meridians as follows: We now interpret a boundary vertex as a shorthand for a vertex with genus weight zero, $r$-weight equal to 1 , but unspecified (arbitrary) euler weight. Thus the isotopy class of a fiber of the corresponding bundle being plumbed is well defined and gives us our meridians, but the isotopy class of a section to the bundle at the boundary is no longer well defined, since it depends on the euler number. The manifold $N^{3}$ obtained by pasting solid tori onto $M(\Gamma)$ as described above is given by the graph $\Gamma^{\prime}$ obtained by deleting the boundary vertices from $\Gamma$.

The calculus for plumbing graph manifolds with meridians (the $M$-calculus for short) is exactly the same as the $F$-calculus except that vertices adjacent to boundary vertices lose their special role. Thus we may blow up or blow down next to boundary vertices and no longer forbid 0-chain absorptions and extrusions of the following type:

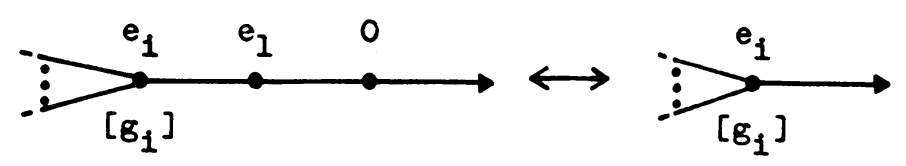

The normal form in this case (called $M$-normal form) is like $F$-normal form except that, again, vertices next to boundary vertices lose their special role. Thus in the situation

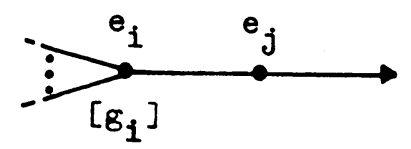

vertex $j$ is considered as part of a chain, so we must have $e_{j} \leqslant-2$ for normal form. There is one exception to this: If vertex $i$ is not on a chain (i.e. it has degree $\geqslant 3$ or $g_{i} \neq 0$ ) we do allow $e_{j}=0$ in normal form, and moreover in this case $e_{i}$ is irrelevant and should be disregarded or set equal to zero.

THEOREM. Any decorated plumbing graph can be reduced by the M-calculus to a unique graph in M-normal form. Any graph manifold with meridians is of the form $M(\Gamma)$ for a unique (up to isomorphism) decorated plumbing graph $\Gamma$ in $M$-normal form.

Again we omit the proof

3. An example: toral links. The graph

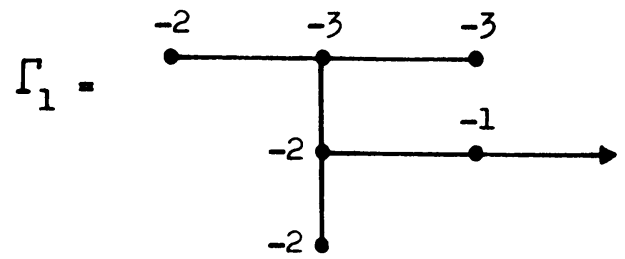


is in $F$-normal form (the $(-1)$-weighted vertex cannot be blown down since it is adjacent to a boundary vertex), so it is the normal form graph for a graph manifold with boundary framing.

If we consider it as the graph of a graph manifold with meridians, then the $(-1)$-weighted vertex can be blown down, and the $M$-normal form is

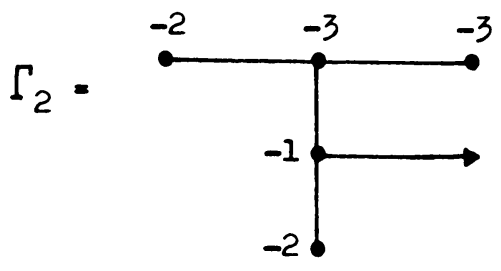

Finally if we forget all boundary structure, the normal form is

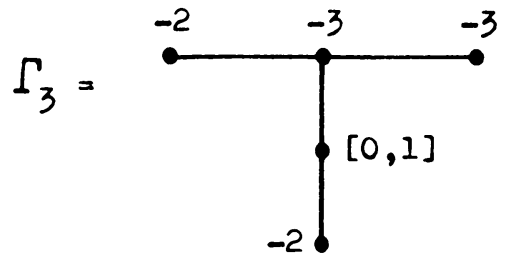

Observe that if one deletes the boundary vertex in $\Gamma_{2}$ then the resulting graph blows down completely, so it represents $S^{3}$. Thus $\Gamma_{2}$ can be considered to represent a knot in $S^{3}$. The knot in question is in fact

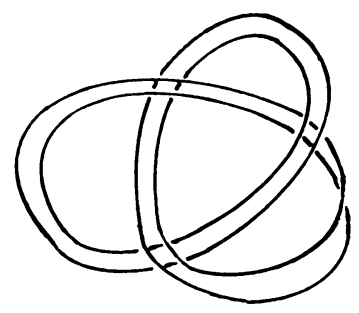

Moreover, the graph $\Gamma_{1}$ thus represents a framing of this knot $K$ (in fact it is the framing in which a longitude links $K$ with linking number 27 in $S^{3}$ ).

More generally if $\Gamma^{\prime}$ is any plumbing graph with $M\left(\Gamma^{\prime}\right)=S^{3}$ and $\Gamma$ is $\Gamma^{\prime}$ decorated with arrows at some vertices, then $\Gamma$ represents a link $K \subset S^{3}$ such that the complement of a tubular neighborhood is a graph manifold. Thus $M$-normal form of such graphs $\Gamma$ classify such links. This is studied in detail in [4]. A special case is iterated torus links, which include all links of plane curve singularities. These are studied in [3]. The explicit example above is of this type. ( $F$-normal forms of such graphs classify framings on such links, but framings on links can be dealt with more easily.)

4. Waldhausen's graphs. One can also define $W$-graphs for boundary framed graph manifolds and graph manifolds with meridians and the analog of Theorem 5.6 then holds. 
For boundary framed graph manifolds the relevant graphs are like Waldhausen's, but decorated with arrows attached at some vertices. The arrows carry no weights. In the situation

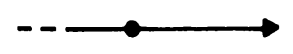

the vertex shown is allowed to have a weight of the form $(0,0, s)$ (such a weight on a vertex of degree 2 is disallowed otherwise by the reduction criteria).

For graph manifolds with meridians the graphs are as above, but arrows also carry weights $(\alpha, \beta)$, with $(0,1)$ permitted, and vertices of degree two never have weight $(0,0, s)$.

In both cases the homomorphism $\varepsilon_{G}: H_{1}\left(G^{*}\right) \rightarrow \mathrm{Z} / 2$ must be modified to a homomorphism $H_{1}\left(G^{* *}, B\right) \rightarrow \mathrm{Z} / 2$, analogously to the case of plumbing graphs.

The translation of Theorem 5.6 to these situations is clear, and is left to the reader.

\section{REFERENCES}

1. F. Bonahon and L. Siebenmann, Les noeuds algébriques (in preparation).

2. P. E. Conner and F. Raymond, Injective actions of toral groups, Topology 10 (1971), 283-296.

3. D. Eisenbud and W. Neumann, Fibering iterated torus links, preprint, 1978; rev. ed., Toral links and plane curve singularities (in preparation).

4. Graph links (in preparation).

5. H. Grauert, Über Modifikationen und exceptionelle analytische Raumformen, Math. Ann. 146 (1962), 331-368.

6. F. Hirzebruch, Hilbert modular surfaces, Enseign. Math. (2) 19 (1973), 183-281.

7. F. Hirzebruch, W. D. Neumann and S. S. Koh, Differentiable manifolds and quadratic forms, Math. Lecture Notes, vol. 4, Dekker, New York, 1972.

8. M. Inoue, New surfaces with no meromorphic functions. II, Complex Analysis and Algebraic Geometry, A Collection of Papers Dedicated to K. Kodaira, (W. L. Baily, Jr. and T. Schioda, eds.), Cambridge Univ. Press, Tokyo and Cambridge, 1977, pp. 91-106.

9. U. Karras, Klassifikation 2-dimensionaler Singularitäten mit auflösbaren lokalen Fundamentalgruppen, Math. Ann. 213 (1975), 231-255.

10. R. Kirby, Problems in low dimensional manifold theory, Proc. Sympos. Pure Math., vol. 32, Amer. Math. Soc., Providence, R. I., 1978, pp. 351-365.

11. _ A calculus for framed links in $S^{3}$, Invent. Math. 45 (1978), 35-56.

12. K. Kodaira, On compact analytic surfaces. II, Ann. of Math. (2) 77 (1963), 563-626.

13. W. D. Neumann, $S^{1}$-actions and the $\alpha$-invariant of their involutions, Bonner Math. Schriften, vol. 44, Bonn, 1970.

14. __ Fibering graph manifolds (in preparation).

15. __ An invariant of plumbed homology spheres, Proc. Internat. Topology Sympos. (Siegen,

1979), Lecture Notes in Math., vol. 788, Springer-Verlag, Berlin and New York, 1980, pp. 125-144.

16. W. D. Neumann and F. Raymond, Seifert manifolds, plumbing, $\mu$-invariant and orientation reversing maps, Proc. Alg. and Geom. Topology (Santa Barbara, 1977), Lecture Notes in Math., vol. 644, Springer-Verlag, Berlin and New York, 1978, pp. 163-196.

17. W. D. Neumann and S. Weintraub, Four-manifolds constructed via plumbing, Math. Ann. 238 (1978), 71-78.

18. P. Orlik, Seifert fiber spaces, Lecture Notes in Math., vol. 291, Springer-Verlag, Berlin and New York, 1972.

19. P. Orlik and F. Raymond, Actions of SO(2) on 3-manifolds, Proc. Conf. on Transformation Groups (New Orleans, 1967), Springer-Verlag, New York, 1968, pp. 297-318.

20. P. Orlik, E. Vogt and H. Zeischang, Zur Topologie gefaserter dreidimensionaler Mannigfaltigkeiten, Topology 6 (1967), 49-64.

21. A. Scharf, Faserungen von Graphenmannigfaltigkeiten, Dissertation, Bonn, 1973; summarized, Math. Ann. 215 (1975), 35-45. 
22. R. Von Randow, Zur Topologie von drei-dimensionalen Baummannigfaltigkeiten, Bonner Math. Schriften, vol. 14, Bonn, 1962.

23. P. Wagreich, Singularities of complex surfaces with solvable local fundamental group, Topology 11 (1971), 51-72.

24. F. Waldhausen, Eine Klasse von 3-dimensionalen Mannigfaltigkeiten, Invent. Math. 3 (1967), 308-333; 4 (1967), 87-117.

25. On irreducible 3-manifolds that are sufficiently large, Ann. of Math. (2) 87 (1968), 56-88.

26. G. B. Winters, On the existence of certain families of curves, Amer. J. Math. 96 (1974), 215-228.

Department of Mathematics, University of Maryland, College Park, Maryland 20742 